\title{
RursuSpicae
}

Transmission, réception et réécriture de textes, de I'Antiquité au Moyen Âge

2 | 2019

Le Physiologus. Manuscrits anciens et tradition médiévale

\section{Barthélemy l'Anglais connaît-il le Physiologus ? État des lieux}

Did Bartholomew the Englishman know the Physiologus? A Survey

\section{Elisa Lonati}

\section{(2) OpenEdition}

Journals

Édition électronique

URL : http://journals.openedition.org/rursuspicae/873

DOI : $10.4000 /$ rursuspicae. 873

ISSN : 2557-8839

Éditeur :

Université Nice-Sophia Antipolis, IRHT - Institut de recherche et d'histoire des textes

Référence électronique

Elisa Lonati, « Barthélemy l'Anglais connaît-il le Physiologus ? État des lieux », RursuSpicae [En ligne], 2 | 2019, mis en ligne le 20 décembre 2019, consulté le 29 mars 2021. URL : http://

journals.openedition.org/rursuspicae/873; DOI : https://doi.org/10.4000/rursuspicae.873

Ce document a été généré automatiquement le 29 mars 2021.

RursuSpicae 


\title{
Barthélemy l'Anglais connaît-il le Physiologus? État des lieux
}

\author{
Did Bartholomew the Englishman know the Physiologus? A Survey
}

\author{
Elisa Lonati
}

${ }^{1}$ Cet article s'ouvre avec une révision critique de quelques aspects de la tradition manuscrite du De proprietatibus rerum de Barthélemy l'Anglais, afin d'y appuyer l'édition provisoire du petit groupe d'extraits à mettre en relation avec leur source, le Physiologus. Le développement des études philologiques abordées par les éditeurs les plus récents nous fournit sous une forme fiable des extraits des livres XII et XVIII, pas encore disponibles à l'heure actuelle en édition critique, afin que nous puissions entreprendre, dans la seconde partie de notre étude, plus ample, une analyse détaillée de leurs sources, problématiquement liées à la "galaxie-Physiologus». La transmission et les évolutions de ce traité bref et ancien de zoologie, minéralogie et botanique allégoriques ont en effet été complexes; elles ont débouché sur la genèse de plusieurs versions anciennes et d'un ample groupe de bestiaires tardifs, dont les rapports mutuels sont encore en cours d'étude. Le fait que le De proprietatibus se nourrisse de plusieurs inspirations combinées, provenant tantôt de l'un ou de l'autre bestiaire, tantôt de compilations qui avaient exploité autrement l'héritage ancien, fait de l'ouvrage de Barthélemy un épisode inédit de la postérité du Physiologus, d'autant plus fascinant qu'il soumet ses notices à un travail systématique de réélaboration et de réécriture.

\section{1. Étude philologique des citations liées au Physiologus dans le cadre d'une tradition complexe}

Le De proprietatibus rerum (désormais DPR), composé par le franciscain Barthélemy l'Anglais autour de 1230-1248 d'abord à Paris, puis continué à Magdebourg, a été pensé à l'origine comme vademecum de philosophie naturelle en dix-neuf livres, à l'usage des élèves du studium de Magdebourg, que l'auteur dirigea à partir de l'année $1231^{2}$. 
L'indiscutable capacité à équilibrer ancien et moderne dans une structure efficace en a déterminé le succès notable, marqué par la diffusion européenne d'un nombre élevé de manuscrits complets et partiels (environ 200 témoins manuscrits conservés et une quinzaine d'éditions anciennes de 1470 à 1609), un éventail énorme de réutilisations et de réécritures (en particulier moralisées) et une dizaine de versions vernaculaires. Un catalogue exhaustif des témoins de la forme originaire du DPR et de celles remaniées, accompagné par une analyse détaillée de leurs caractéristiques macrotextuelles et de leur distribution par région et par époque a été dressé par $\mathrm{H}$. Meyer ${ }^{3}$. La première édition critique du texte original est en cours chez Brepols sous la direction de B. Van den Abeele, Chr. Meier-Staubach et H. Meyer et la coordination de I. Ventura ${ }^{4}$. Plusieurs contributions se sont penchées dans les dernières décennies sur les vulgarisations du $D P R$, pour en étudier les modalités de traduction et le type de modèle latin utilisé5. Dans l'attente de la publication intégrale de l'édition critique collective, pour une grande partie du texte il est encore nécessaire d'utiliser la dernière des éditions anciennes, publiée en 1601 à Francfort et réimprimée plusieurs fois ${ }^{6}$. Il reste à mener une enquête plus approfondie sur les rapports existant entre les témoins de la tradition directe, afin de mettre en évidence les codices les plus importants du point de vue stemmatique.

Étant donné l'ampleur de cette tradition, les éditeurs modernes ont fondé leur texte sur un nombre restreint de témoins, choisis en raison de leur qualité afin de publier une édition «acceptable» du point du vue philologique. Une dizaine de codices potentiellement intéressants ont été tout d'abord identifiés sur la base d'une série de critères, à savoir le fait qu'ils soient complets, leur proximité pour la plupart du milieu de l'université parisienne (où la circulation du texte fut précoce) et le fait qu'ils témoignent de la première phase de diffusion du texte (le critère de la datation aurait été peu utile, car il est difficile, voire impossible, de retrouver des témoins manuscrits proches de la date de composition). Les codices ont été collationnés par sondages (un chapitre par livre), ce qui a conduit à la sélection finale de cinq témoins comme base pour la constitutio textus ${ }^{7}$ :

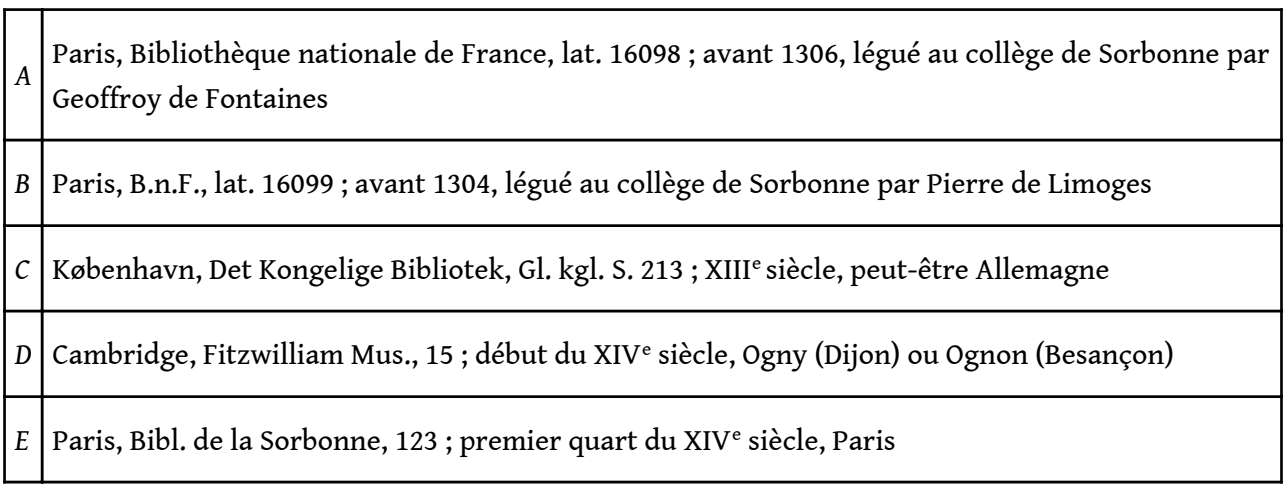

Les éditeurs ont donné quelques indications générales sur le comportement de ces témoins, en signalant notamment qu'il y a une relation étroite entre $A$ et $B$, les deux exemplaires les plus anciens du DPR, alors que les manuscrits $D$ et $E$ sont assez tardifs et corrompus; de nombreuses corrections importantes ont été apportées dans $B$ par une main correctrice contemporaine, siglée $B^{2}$, et ces leçons se retrouvent souvent dans $C$, un témoin de provenance allemande particulièrement correct. Comme aucune enquête n'a été conduite pour placer les cinq manuscrits dans un stemma, l'édition critique s'est fondée surtout sur $C$ et $B^{2}$; un essai à été fait plus récemment par R. Casapullo, mais 
n'ayant pas remarqué que $D$ et $E$ sont des témoins contaminés, elle a proposé une évaluation en partie incorrecte de leur comportement ${ }^{8}$. D'ailleurs, la tradition du DPR est tellement compliquée qu'un stemma traditionnel est pour l'instant impossible à tracer.

\subsection{Quelques nouvelles considérations méthodologiques sur la tradition du $D P R$}

À partir des reprises que Barthélemy a tirées de la Naturalis historia de Pline l'Ancien, j'ai conduit pour mon mémoire de fin d'études une révision des matériaux publiés en 2007 et abordé l'étude d'autres sections encore dépourvues d'une édition moderne, en tenant compte des manuscrits A B C D E et de seize nouveaux exemplaires, de datation et de provenance diversifiées, choisis en raison de leur accessibilité (ils sont tous disponibles en ligne) ${ }^{9}$. L'absence presque complète d'indices sur la tradition du DPR autorise de premiers sondages même sur des témoins tardifs et corrompus, d'autant plus qu'ils se caractérisent par un ensemble de phénomènes qui en font un échantillon représentatif d'un plus grand nombre de codices. Il s'agit notamment de :

\begin{tabular}{|c|c|}
\hline$F$ & Einsiedeln, Stiftsbibliothek, 299 ex. 192 ; XIV ${ }^{\mathrm{e}}$ siècle, abbaye bénédictine d'Einsiedeln \\
\hline G & $\begin{array}{l}\text { Firenze, Biblioteca Medicea Laurenziana, Plut. XVIII sin. } 9 \text {; XIV }{ }^{\mathrm{e}} \text { siècle, couvent franciscain de } \\
\text { Santa Croce, Florence }\end{array}$ \\
\hline$H$ & Firenze, B.M.L., Plut. XXI sin. 2 ; XIV e siècle, couvent franciscain de Santa Croce, Florence \\
\hline$I$ & Firenze, B.M.L., Plut. XXI sin. 3 ; XIV ${ }^{\mathrm{e}}$ siècle, couvent franciscain de Santa Croce, Florence \\
\hline$J$ & Leipzig, Universitätsbibliothek, $1424 ; 1457$, Frankfurt-am-Main \\
\hline K & Leipzig, Universitätsbibliothek, 1425 ; XIV siècle \\
\hline$L$ & Madrid, Biblioteca Nacional, 930 ; XIV siècle, cathédrale de Messine (Italie) \\
\hline$M$ & Madrid, B.N., 3316 ; XIV siècle \\
\hline$N$ & $\begin{array}{l}\text { Montpellier, Bibliothèque interuniversitaire de Médecine, Faculté de Médecine, H } 189 \text {; XIV } \\
\text { siècle, abbaye cistercienne de Clairvaux }\end{array}$ \\
\hline 0 & Montpellier, B.IU.M., Faculté de Médecine, H 190 ; XIV e siècle, abbaye cistercienne de Clairvaux \\
\hline$P$ & Paris, B.n.F., lat. 347 ; c. 1300 , cathédrale de Châlons-sur-Marne \\
\hline$Q$ & Paris, B.n.F., lat. 347E ; XIV ${ }^{e}$ siècle \\
\hline$R$ & $\begin{array}{l}\text { Praha, Narodni Knihovna, I.C. } 34 ; 1475 \text {, abbaye augustinienne de Trebon (Tchéquie, Bohême } \\
\text { méridionale) }\end{array}$ \\
\hline$S$ & Reims, Bibliothèque municipale, $992 ; 1325$, abbaye bénédictine de Saint-Rémi, Reims \\
\hline$T$ & Toulouse, Bibliothèque municipale, 225 ; XIV ${ }^{\mathrm{e}}$ siècle, abbaye augustinienne de Toulouse \\
\hline
\end{tabular}


Troyes, Bibliothèque municipale, $979 ; \mathrm{XIV}^{\mathrm{e}}$ siècle, abbaye cistercienne de Clairvaux

Ces travaux ont débouché sur un placement progressif de chaque manuscrit en relation avec les autres et sur l'édition critique d'une série d'extraits des livres XII, XIII, XVI et XVIII, accompagnés par des conclusions générales possiblement valides pour une section plus ample de la tradition du DPR. À la lumière de ces conclusions, et sur la base des collations menées pour éditer ci-dessous les extraits du DPR liés au Physiologus, nous allons donc proposer quelques observations préliminaires, qui présentent les phénomènes majeurs de cette tradition et expliquent quels critères ont été suivis dans la préparation de notre édition provisoire ${ }^{10}$.

Une première question est de savoir s'il existe ou pas un ancêtre commun à cette vingtaine de témoins. Bien que choisis de façon aléatoire, nos codices partagent quelques erreurs, ce qui signifie qu'ils remontent à un seul ancêtre, qui pourrait être l'archétype de toute la tradition du DPR ou seulement un subarchétype. Étant donné que, dans ces cas, l'édition de 1601 offre des leçons correctes trop difficiles pour être atteintes ope ingenii, l'hypothèse est qu'elles proviennent d'exemplaires du DPR faisant partie d'autres branches de la tradition et que les témoins qui les conservent (comme $H$ et $P$ ) auraient subi une contamination.

XVIII 14 robore suo $\mathrm{H}$ ed. : robora $A B C D E G I K L M N O P Q R S T U$ : robore $P^{2}$

XVIII 43 querit HP ed. : comedit ENS : reperit $T^{2}$ : om. ABCDGIKLMOQRTU

XVIII 76 duodecies in die $\mathrm{H}$ ed. : duodecies ABDEGIJKLMNOPQRSTU : duodenes $C$

XVIII 76 vividus ed. : invidus ABDEGHIKLMNOPQRSTU : idus $C$ : mundus $D^{2}$

Les résultats d'un phénomène de contamination massive et stratifiée se manifestent en effet dans la plus grande partie de nos codices, d'une manière variable selon le manuscrit ou le groupe de manuscrits : cela signifie qu'un nombre plus ou moins élevé d'erreurs héritées des ancêtres a été corrigé, et que quelques variantes incorrectes ont été ajoutées, sur le modèle du travail fait dans $B$ par $B^{2}$. Comme pour $B^{2}$, il n'est pas facile de dire de quelle partie de la tradition manuscrite ces leçons découlent, mais elles se retrouvent déjà dans le texte principal de nos témoins et, bien qu'évidemment contaminées, elles ne sont pas toujours les mêmes dans tous les manuscrits. Il nous semble possible de confirmer l'évaluation des éditeurs quant à $C$, un témoin apparemment non contaminé et isolable non seulement de $A B D E$, mais aussi des autres seize codices. Une fois exclu $C$, tous les autres témoins feraient partie de la même branche, mais il est impossible de dresser un stemma: on se contente d'identifier un texte « de base » que tous partagent, caractérisé par une série d'erreurs significatives, à partir duquel on peut évaluer le degré plus ou moins haut de contamination de chaque témoin. Le texte " de base ", particulièrement fautif et apparemment non contaminé, se trouve dans $A$ et $B$, auxquels on ajoute $M$ et $U$ et, pour la plupart du texte, aussi $H L F J$ : les deux premiers récupèrent toutefois à certains endroits des leçons correctes à travers la contamination, alors que $F$ et $J$ sont si lacunaires que nous les avons rarement à notre disposition. Comme les erreurs de $A B$ se trouvent aussi dans ces témoins, leur relation est moins exclusive qu'on ne l'a suggéré dans l'édition de 2007.

XVIII 43 facta] femina $A B^{a c} H L M U$ : femora $F$

XVIII 95 ad navem...perferens] ferens $A B^{a c} H M U$

Parmi les manuscrits contaminés d'une façon limitée, chacun pour des leçons différentes, il y a $R I K$, qui évitent une partie des erreurs des manuscrits proches de $A B$ et présentent quelques leçons correctes propres à $C$. Plus contaminés sont $P$ et surtout 
$Q$, qui évite un nombre élevé d'erreurs du texte "de base » et récupère une variante incorrecte ajoutée dans $B$ par $B^{2}$. Cela arrive plus fréquemment encore dans $O T$ et $D$, mais le texte principal de ce dernier présente souvent des monstra : il s'agit de leçons doubles, dont l'une est l'erreur du texte «de base » et l'autre la correction atteinte à travers la contamination. Un témoin particulier est $G$, qui, bien que très fautif et proche du texte "de base ", conserve ici et là quelques leçons correctes et quelques variantes incorrectes extrêmement rares, propres seulement à $B^{2}$ ou à des témoins très contaminés. Voici ci-dessous un petit échantillon qui décrit ces phénomènes :

XVIII 14 complexas] perplexas $B^{2} O Q T$ : per complexas $D$

XVIII 43 inclinant $D E G N Q S T$ ed. : inclinat $J$ : includunt $C I O$ : includant $P$ : inclusant $B^{2}$ :

includit $A B F H K L M R U$

XVIII 69 loca $B^{2} C D E G N O Q S T$ ed. : locum $H$ : littus ABFIKLMPRU

XVIII 76 XXV B²DEGLNOPST ed. : XLV ABCHIKMQRU

XVIII 76 diversificant] vivificant $C$ : vel diiudicant in $\operatorname{marg} . B^{2}$ : diiudicant $G \mid$ attrahit]

trahit $B^{2} G$

XVIII 95 dolet $B^{2} D E G L N O P Q S T$ ed. : dicitur dolet $B C$ : dicitur $M$, A spat. rel. : dolere

dicitur $I$ : dicitur turbari $R$ : dicitur contristari $K U$ : tristatur $H J$

XVIII 101 qui sollicite CGIKR: qui sollite $J$ : qui absolute ABDELMNOPQSTU: que

absolute $e d$ : qui $F$ : que $H$

Enfin, la présentation de $E$ offerte par l'édition de 2007 exige une révision. En fait, ce témoin n'est pas isolé, car il partage un certain nombre d'erreurs avec $N$ et $S$, et les trois sont contaminés d'une façon importante, ce qui signifie que leur texte est souvent plus correct qu'on ne l'a dit et mérite d'être pris en considération.

XVIII 14 ligaminibus] luctaminibus ENS

XVIII 43 querit HP ed. : comedit ENS : reperit $T^{2}$ : om. ABCDGIKLMOQRTU

XVIII 43 pro viribus post protegit $E$, ante NS

XII 22 homo ille EGNS : ille homo $R$ : homo ACDFHIJKLMOPQTU ed.

Pour résumer, bien qu'ils soient les témoins les plus anciens du DPR, $A$ et $B$ transmettent un texte très corrompu et évidemment loin de l'original, dont certaines erreurs sont répandues dans plusieurs témoins, alors que d'autres ont été corrigées à travers la contamination. Ce phénomène empêche pour l'instant de définir les rapports entre les codices, mais il a significativement amélioré le texte transmis par $A B$, en récupérant de bonnes leçons à partir de sources inconnues, de grande qualité et proches de l'original. Si l'on veut établir un texte le plus possible correct, les codices très contaminés doivent nécessairement être consultés en comparaison avec $C$ et $B^{2}$, en sachant que, à certains endroits, ils sont supérieurs même aux deux témoins sur lesquels l'édition de 2007 a fondé sa constitutio. À côté de $E$, il est important de regarder au moins $O T Q$ et occasionnellement $G$; quant à $D$, il pourrait être écarté, car il transmet des doubles leçons non originelles, dont la partie correcte peut être récupérée de manuscrits contaminés de qualité supérieure ${ }^{11}$.

\section{2 Édition des extraits du DPR liés au Physiologus}

À la lumière des considérations illustrées ci-dessus, nous présentons une édition critique provisoire des citations du DPR en référence à la source Physiologus, à savoir dix extraits provenant du livre XVIII, que le DPR lui attribue explicitement, et six autres passages dont l'attribution est (apparemment) problématique. L'ensemble des réflexions que les extraits suscitent fera l'objet de la section suivante, qui se consacre à 
l'identification des sources directes et indirectes à travers lesquelles Barthélemy a eu accès à ces informations.

XVIII 8 De angue. Modus autem renovationis ipsius anguis satis videtur mirabilis. Nam, ut dicit Physiologus, anguis se sentiens morbo vel senio aggravatum pluribus diebus abstinet a cibo et ieiunat, ut sic pellis eius a carne facilius relaxetur; deinde, gustata herba quadam amara, vi herbe provocatur ${ }^{1}$ ad vomitum, et sic evomit humorem virulentum, qui fuit causa sue infirmitatis et defectus. Tandem, ut cutem rigidam temperet et mollificet, in aqua se balneat ac humectat, et sic angustam rimam petre alicuius ${ }^{2}$ seu cavernam querens per rime angustiam intrat et, cum quadam violentia transiens, ab exuvia penitus se decorticat ac denudat. Et tandem soli expositus se desiccat et in carnis superficie $e^{3}$ novam cutem recuperat, sumptis viribus videt clarius ${ }^{4}$, incedit ${ }^{5}$ ac repit fortius ac comedit avidius quam ante depositionem exuvie faciebat.

1. herbe provocatur $B^{2} C E G N P^{2} Q S$ ed. herbe provocetur DLOPT: herba provocetur $A B I K M R U$ : provocatur $F H$ : provocetur $J \mid 2$. petre alicuius] alicuius petrae ed. : om. $Q \mid$ 3. superficie] superficiem CDIKR |4. videt clarius] clarius videt $e d$. $\mid 5$. incedit] insedit $B^{2}$

XVIII 28 De castore. Unde quod dicit Isidorus et Physiologus de eorum castratione non de usualibus fibris, sed de aliquibus aliis animalibus, que castoribus assimilantur ${ }^{1}$ in testiculis, est forsitan intelligendum.

1. que castoribus assimilantur $B^{2} C D^{2} E G N O Q S T$ ed.: assimilantibus ABDFIKLMPRU: illis similibus $H$

XVIII 32 De cocodrillo. Dicit autem Physiologus: Cocodrillus ${ }^{1}$, si quendam ${ }^{2}$ invenit

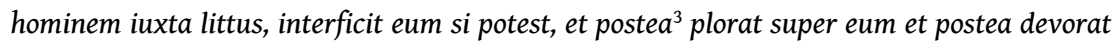
ipsum. Et dicit quod de eius fimo fit unguentum, unde facies mulierum sophisticantur, ut senes et rugose iuvencule per tempus videantur. Herbas bonas ${ }^{4}$ libenter comedit, inter quas enidros ${ }^{5}$ serpens parvulus, qui ei inimicatur, caute se involvit; et dum cocodrillus herbas carpit, serpentem transglutit, qui intrans ventrem eius omnia interiora carpit, et sic interimit eum et occidit et sic illesus exit.

1. cocodrillus] crocodilus ed. $\mid$ 2. si quendam ed. : siquidem $A B C D H L M O P Q T$ : siquidem si EKNS : quidem si $U$ : si quem $R$ : si quemquam $I$ : inquid $G \mid$ 3. postea $B D^{2} E N O S T U$ : post CGKR: preterea $A D L M P Q$ : prius $I$ : primo ed. om. $H \mid$ 4. bonas om. ed.|5.enidros] enhydris ed.

XVIII 43 De elephantibus. In libro autem Physiologi de elephante memini me sic legisse: Elephas, inquit, est animal quod magnitudine inter ${ }^{1}$ omnia animalia quadrupedia preexcellit, intellectu et memoria multum vigens. Nam elephantes inter alia eorum facta ${ }^{2}$ nunquam dormiendo totaliter se inclinant ${ }^{3}$; quando fessi sunt arbori et maxime palme quietis gratia accubant, et sic qualitercunque se sustentant. Quorum quieti homines insidiantes occulte arborem concavant, cui se appodians elephas et nesciens fraudem pondere corporis arborem frangit, et frangendo cadit subito et succumbit. Qui casum suum videns irrecuperabilem ${ }^{4}$ miro modo barrit, id est clamat et rugit; ad cuius barritum multitudo iuvenum subito accedit elephantum, qui paulatim seniorem pro viribus elevant et, ut eum relevent ${ }^{5}$, miro affectu se totis viribus inclinant ${ }^{6}$. Coitum abhorrent nisi pro sola prole, unde dicitur ibidem quod, quando ${ }^{7}$ elephantes Indicos stimulat ${ }^{8}$ vis ${ }^{9}$ amoris, femina precedit versus orientem, quam sequitur masculus, quousque in abscondito deveniant ${ }^{10}$. Qualitercunque poterit ${ }^{11}$ mandragoram querit ${ }^{12}$, cuius fructum primo femina gustat, deinde masculus eundem fructum comedens eam impregnat et fecundat ${ }^{13}$, ut dicitur. Sed diu propter magnitudinem fetus femina in utero fetum gerit; sed tempore partus in aquis et in insulis fetum procreat propter draconis metum, ne ipsum tenellum fetum sorbeat aut deducat; et dum mater filium parturiendo laborat, masculus pro viribus ${ }^{14}$ ipsam protegit et defendit. Item dicitur ibidem quod ossa elephantis combusta fugant serpentes et omnia venenosa. Item aliud dicitur ibidem valde admirandum: dicit enim quod apud Ethiopes in aliquibus regionibus sic venantur. Accedunt ad deserta, ubi ${ }^{15}$ due virgines omnino nude habitant et crinibus resolute, quarum una fert alveolum, alia gladium; incipiunt autem alte cantare, quarum 
cantum audiens bestia venit ad eas et lingit earum mammas. De dulcedine autem cantus mox obdormit elephas, et tunc una perforat gladio guttur ${ }^{16}$ vel $^{17}$ latus ${ }^{18}$, altera vero colligit sanguinem eius quo tingitur pannus, qui purpura appellatur.

1. inter DENOQST ed. : om. ABCGHIJKLMPU : sua $R \mid 2$. facta] femina $A B^{a c} H L M U$ : femora $F \mid$

3. inclinant $D E G N Q S T$ ed. : inclinat $J$ : includunt $C I O$ : includant $P$ : inclusant $B^{2}$ : includit ABFHKLMRU | 4. irrecuperabilem FHI : irreparabilem ed. : irrecuperabiliter $A B C D E G J K L M N O P Q R S T U$ | 5. relevent] elevent EGNS | 6. se...inclinant DENOQST ed. : se inclinant $A B F G H I J K L M P R U$ : se inclinat $C \mid$ 7. quando om. $A B^{a c} I K L M U \mid$ 8. stimulat] luxuria stimulat ed. | 9. vis ENPRS : vi ABDKLMOQTU ed. : in CI : vix G|10. deveniant DENOPQS ed. : veniant $A B H T$ : inveniant $B^{2} C I K R U$ : inveniatur $M$ : inveniat $G L \mid 11$. poterit] poterint $B R T^{a c} U$ : poterunt $C$ : potuerint $G$ : poterant $I \mid 12$. querit $H P$ ed. : comedit $E N S$ : reperit $T^{2}$ : om. ABCDGIKLMOQRTU | 13. et fecundat post ut dicitur CGIKR | 14. pro viribus post protegit $E$, ante NS $\mid 15$. ubi ante habitant CFIJRU $\mid 16$. guttur] eius guttur EHNS : guttur eius $J \mid$

17. vel] et EFJRS | 18. latus] latus eius $B^{2} C G O T$ : latus ipsius $F$

XVIII 69 De lupo. De lupis autem dicit Physiologus: Virtus luporum in pectore et in unguibus viget ac in ore, in posterioribus vero minime. Collum retroflecter $e^{1}$ non valet in ${ }^{2}$ nullo mense nisi in Maio, quando fiunt tonitrua. Cuius astutia est ut non capiat predam iuxta loca ${ }^{3}$ ubi nutrit fetus suos, sed tunc ${ }^{4}$ temporis venatur a remotis. Quando autem noctu $u^{5}$ prede gratia pergit ad ovile, ne canes eius sentiant odorem vadit contra ventum; et si aliquo casu pes eius calcando super aliquid strepitum fecerit, ipsum pedem castigat duro morsu. Oculi eius lucent de nocte ut lucerne et, ut dicit Solinus, villum ${ }^{6}$ amoris excitativum in cauda portat, quod dentibus evellit quando timet capi.

1. retroflectere $B^{2} C D E N O S T$ ed.: reflectere $A B G H I K L M P Q R U \mid 2$.in] et in ed. | 3. loca $B^{2} C D E G N O Q S T$ ed.: locum $H$ : littus ABFIKLMPRU| 4. sed tunc $B^{2} E G N S T$ : et tunc ABDIKMOPRU ed. : tunc $C Q$ : sed $F$ om. temporis : tunc enim $H:$ om. $L \mid 5$. noctu] nocte $F R$ ed. | 6. villum $B^{2} C D E F G I N O Q S T$ : vultum $A B U$ : multum $D^{2} K^{2} L M P R$ ed. : sunt $H$

XVIII 76 De onagro. De quo dicit Physiologus quod XXV1 die Martii duodecies in die ${ }^{2}$ et totiens in nocte rugit, per cuius rugitum ${ }^{3}$ equinoctium apud Affros discernitur, et dicit quod totiens semper rugit de die quot dies habet horas ${ }^{4}$, similiter et de nocte. Unde et sylvani ${ }^{5}$ in montibus Affrice, in quibus onagri ${ }^{6}$ abundant, per ${ }^{7}$ numerum rugitus eorum numerum horarum, dierum et noctium computant et diversificant ${ }^{8}$. Sagax et vividus ${ }^{9}$ est in olfactu, unde quando amore fervet et nescit ubi eius femina ${ }^{10}$ evagatur, rupem ascendit et patulis naribus ventum attrahit ${ }^{11}$; per cuius flatum ubi sit eius femina diiudicat et discernit. In montibus altis ${ }^{12}$ pascuosis herbas virentes, quas multum diligit, cum diligentia querere consuevit, quas cum invenerit pre gaudio statim rudit; et ubi presenserit bestiam vel hominem ipsum venari cupientem, quamdiu invenit ${ }^{13}$ gramina virentia a pascuis ${ }^{14}$ non recedit. Hominum frequentiam valde odit atque fugit, solitudines et deserta multum diligit.

1. XXV B BEGLNOPST ed.: XLV ABCHIKMQRU| 2. duodecies in die $H$ ed.: duodecies ABDEGIJKLMNOPQRSTU: duodenes $C \mid$ 3. rugitum om. $A B^{a c} I J K M R \mid$ 4. dies habet horas] horas habet dies EHNS : horas dies habet $R$ : habet horas dies $J \mid 5$. sylvani $B^{2}$ ed. : silvam $A B C D E G H I K L M N O P Q R S T U$ | 6. in quibus onagri $B^{2} C E G N S$ : onagri in quibus $A B D L M O P Q T U$ : ab onagris in quibus $e d$. quibus onager $R$ : onagri in $H$ : onagri $K$ : in quibus $I \mid 7$. per $B^{2} C G$ ed. : om. ABDEIKLMNOPQRSTU | 8. diversificant] vivificant $C$ : vel diiudicant in marg. $B^{2}$ : diiudicant $G \mid 9$. vividus ed. : invidus $A B D E G H I K L M N O P Q R S T U$ : idus $C$ : mundus $D^{2} \mid$ 10. eius femina] femina eius EFS : femina est $N$ : est femina $R \mid 11$. attrahit] trahit $B^{2} G \mid$ 12. altis] altis et $O$ ed. $\mid 13$. invenit] invenerit EFLNS : evit $0:$ om. $M, Q$ spat. rel. | 14. a pascuis] de pascuis ENS : pascuis $O Q$

XVIII 77 De onocentauro. Sed alio modo sentit Physiologus, qui dicit onocentaurum esse compositum ex humana effigie et asinina. Nam ab umbilico et sursum figuram habet hominis et ab eodem inferius formam obtinet animalis. 
XVIII 80 De panthera. De panthera dicit autem ${ }^{1}$ Physiologus: Panthera, inquit ${ }^{2}$, odit draconem et draco ipsum fugit. Cum autem comederit et saturatus ${ }^{3}$ fuerit, se recondit in spelunca et dormit continue fere per $^{4}$ tres dies; post triduum vero, a somno surgens, emittit vocem et ab ore eius ${ }^{5}$ exit odor aromaticus supra modum suavis, propter cuius suavitatem ipsum omnia animantia sequuntur. Solus autem draco, audiens vocem eius, timore perterritus fugit in cavernam, nec ferens odorem in semetipso deficit et torpescit: odorem enim eius reputat pro veneno.

1. autem om. $F$ ed. | 2. inquit om. $A B^{a c} C H K M R U \mid$ 3. saturatus conieci : saturata codd., ed. |

4. per om. ed. 5 . ore eius] eius ore $F$ ed. : ore $U$

XVIII 95 De syrena. De syrena autem ${ }^{1}$ dicit Physiologus: Syrena ${ }^{2}$ est monstrum marinum ab umbilico et sursum habens ${ }^{3}$ formam virginis, inferius figuram piscis. Hec belua in tempestate gaudet, in sereno autem dolet ${ }^{4}$. Hec dulcedine cantus facit dormire navigantes, quos cum viderit consopitos ad navem accedit et quem poterit rapere secum ducit. Et perferens ${ }^{5}$ ipsum ad locum siccum ${ }^{6}$, primo ${ }^{7}$ ipsum secum coire cogit; quod si noluerit ${ }^{8}$ vel non poterit, ipsum ${ }^{9}$ perimit et eius carnes devorat et transglutit. De talibus monstris legitur in Historia Magni Alexandri ${ }^{10}$.

1. syrena autem] syrena $C R$ : sirene autem ed. 2 . syrena] siren ed. 3 . habens ante ab umbilico CGHIKMRU | 4. dolet $B^{2} D E G L N O P Q S T$ ed. : dicitur dolet $B C$ : dicitur $M$, A spat. rel. : dolere dicitur $I$ : dicitur turbari $R$ : dicitur contristari $K U$ : tristatur $H J \mid 5$. ad navem... perferens] ferens $A B^{a c} H M U \mid$ 6. siccum] suum EJNS | 7. primo] primum ed. | 8. noluerit] coire noluerit ed. | 9. ipsum] illum ed. | 10. Magni Alexandri] Alexandri Magni ed.

XVIII 101 De taxo. Est ${ }^{1}$ quedam species taxi, ut dicit Physiologus, qui sollicite ${ }^{2}$ cibos contra hiemem una cum femina colligit ${ }^{3}$ et reponit; et veniente algore hiemis, timens masculus ne cibi collecti nimis cito deficiant, feminam ab esu reprimit et ipsam ad ${ }^{4}$ saturitatem comedere non permittit. Que pacem simulans et quasi taxo masculo ${ }^{5}$ cedens, cavernam latenter exit et, per partem oppositam latibulum intrans, ignorante masculo fauces aperit et diu ${ }^{6}$ aggregatos cibos devorat et consumit. Hec bestia, ut dicit idem, vulpem odit et cum eo ${ }^{7}$ dimicare consuevit, sed videns vulpes quod propter duritiam villosam ${ }^{8}$ eius pellem ledere $e^{9}$ non poterit, se victum ${ }^{10}$ simulans fugam petit; et dum taxus predam querit, vulpes eius latibulum subintrans urina et aliis immunditiis taxi cubiculum inficere consuevit, cuius fetorem abhorrens melus ${ }^{11}$ defedatum domicilium derelinquit et aliam mansiunculam necessario ${ }^{12}$ sibi querit.

1. est] est etiam CFIKR : est autem $G \mid 2$. qui sollicite CGIKR : qui sollite $J$ : qui absolute $A B D E L M N O P Q S T U$ : que absolute ed. : qui $F$ : que $H \mid$ 3. colligit] recolligit CGIJKR $\mid 4$. ad] in $A B^{a c} I J K M U:$ ad in $L:$ ne ad $R \mid$ 5. taxo masculo] masculo taxo ENST : masculo $F \mid$ 6. et diu CENRS: diu ABDFGIJKLMOPQTU ed.|7.eo] eadem ed. | 8. villosam $F$ : et villosam $A B C D E G I K L N O P Q R S T$ ed. : et villositatem $U$ : om. $H M \mid 9$. ledere] et ledere $R$ : eum ledere $U$ ed.: ledere eum $J \mid$ 10. victum] victam ed. | 11. melus] melis ed. | 12. necessario] necessariam $R$ ed.

XII 14 De fenice. De hac ave dicit Physiologus ${ }^{1}$ quod fenix est avis sine pari, vivens CCC vel $D$ annis; quibus completis, cum suum sentit defectum, nidum facit ex lignis aromaticis et multum siccis, qui ${ }^{2}$ in estate ex fervore solis flante Favonio accenduntur. Quibus iam accensis, fenix sponte nidum ${ }^{3}$ ingreditur et ibidem inter ligna ardentia incineratur; ex quo cinere infra triduum quidam vermiculus nascitur, qui paulatim plumas recipiens in volucrem reformatur.

1. Physiologus CFGK : Philosophus ADEHILMNOPQRSTU ed. | 2. qui] quae ed. | 3. fenix sponte nidum ADGIJOPRT : fenix nidum sponte $C$ : sponte fenix nidum ENS : sponte nidum FHKLMQU ed.

XII 22 De kaladrio. Kaladrius ${ }^{1}$ secundum Physiologum ${ }^{2}$ est albi coloris, nullam habens partem nigredinis, cuius pars inferior femoris ipsius purgat caliginem oculorum. Cuius natura est talis quod, quando aliquis gravi detinetur infirmitate, si egritudo fuerit ad 
mortem kaladrius avertit faciem suam a sic egrotante, et sine dubio tunc moritur homo ille; si autem infirmus debet convalescere, kaladrius visum figit ${ }^{4}$ in ipsum et intendit in eum quasi applaudens ei.

1. kaladrius] charadrius ed. 2. Physiologum CFGK : Philosophum ADEHILMNOPQRSTU ed. | 3. homo ille EGNS : ille homo $R$ : homo ACDFHIJKLMOPQTU ed. | 4. visum figit] figit visum ed.

XII 29 De pellicano. Et idem dicit Physiologus ${ }^{1}$ per hec verba: Pellicanus ${ }^{2}$, inquit, est nimius ${ }^{3}$ amator filiorum suorum. Cum enim gignit natos et incipiunt crescere, parentes suos in faciem percutiunt, propter quod ipsos mater repercutit et occidit. Tertio vero die mater se in costa usque ad effusionem sanguinis percutit, et calidum sanguinem super corpora mortuorum filiorum ${ }^{4}$ post effundit; ex cuius virtute pullus prius mortuus reviviscit.

1. Physiologus C: Philosophus ADEGHIKLMNOPQSTU: Plinius $R$ ed.|2.pellicanus] pelicanus ed. | 3. nimius] avis ed. | 4. mortuorum filiorum] filiorum mortuorum ENRS

XII 1 De aquila. Ad hoc ${ }^{1}$ dicit Augustinus et Plinius quod aquila in senectute patitur caliginem in oculis et gravedinem in alis suis, contra quod incommodum instruitur a natura ut fontem aque scaturientis querat. Deinde ascendit quantum potest per aera, donec ex calore aeris ${ }^{2}$ et labore volatus ${ }^{3}$ fortius incalescat, unde tunc ex calore poris apertis et pennis relaxatis subito descendens in fontem ruit; $i b i^{4}$ mutatis plumis et purgata caligine in oculis vires recipit et resumit.

1. ad hoc] ad haec ed. | 2. aeris] solis ed. | 3. volatus CDENOPQRST ed.: volatus stomachus AFGHIJKLMU (om. fortius GIJK) | 4. ibi ACDMOPQRTU : ibique ENS ed. : et ibi $\mathrm{J}$ : ubi FGHKL : i I

XVIII 14 De bubalo [...] ut dicit Plinius libro XXVIII capitulo X [...] Est et ${ }^{1}$ aliud animal simile bovi agresti, sed non est tante magnitudinis; maxima tamen habet cornua multum alta et acuta, cum quibus deicit arbores et arbusta; si autem ${ }^{2}$ concava est quercus, robore suo $^{3}$ deponit usque ad terram. Qui gratia pastus tandem ${ }^{4}$ caput submittit inter frutices subtilia et prolixa virgulta sive vimina habentes, quarum complexione et adherentia cornua bestie circumligantur et obligantur, unde diu luctans contra illas complexas ${ }^{5}$ virgultorum obvolutiones magis ac magis se intricat atque ligat; cumque diu luctans ab illis ligaminibus ${ }^{6}$ se non expedit, immo fortius se involvit, pre indignatione alte ${ }^{7}$ mugit. Cuius vocem horridam audiens venator scit bestiam illaqueatam fore et detentam, unde ipsam secure venabulis impetit, et occidit bestiam acerrimam in virgultis, quam in nemoribus magnis invadere nullatenus ausus fuit. Et hanc bestiam vocat Physiologus aptalonem ${ }^{8}$, cuius dictis si fides adhibenda est mirum videtur ${ }^{9}$, qualiter tam fera bestia de virgultis et nemoribus modicis cornua non eximit, que eisdem cornibus ingentes arbores deicit et prosternit.

1. est et $A B C D F G H I K L M P Q R T U$ : est etiam $O$ : est autem et ENS : est autem ed.|2. si autem $C$ : si $H$ : sed et $A B^{2} D G K L M O^{2} P Q T U$ : sed BENOS : sed que $R$ : sed si $I$ : sed et si ed. 3 . robore suo $H$ ed. : robora $A B C D E G I K L M N O P Q R S T U$ : robore $P^{2} \mid$ 4. gratia pastus tandem] tandem gratia pastus $C G H I K R \mid 5$. complexas] perplexas $B^{2} O Q T$ : per complexas $D \mid 6$. ligaminibus] luctaminibus $E N S \mid$ 7. alte om. $A B^{a c} H L M$ : atque $K U \mid$ 8. aptalonem $B^{2} C G I N Q S T$ : aptalenem $E K$ : aptalanem $R$ : aptaleonem ABDHLMOPU ed.: aptoleonem $F$ (cf. Phys. autolops) । 9. videtur] videtur esse ENS : esse videtur HMP

XII 37 De upupa. De hac ${ }^{1}$ dicunt physici $i^{2}$ quod, cum senuerit ita quod ${ }^{3}$ nec videre nec volare queat, pulli eius evellunt ei pennas invalidas et liniunt ei oculos herbarum succis et fovent sub alas ${ }^{4}$, donec recrescant ${ }^{5}$ plume eius et sic renovata perfecte volet et videat ${ }^{6}$ clare sicut et ipsi $^{7}$, ut dicit Isidorus.

1. hac] hac ave ed. $\mid$ 2. physici] philosophi $J \mid$ 3. ita quod] eo quod $A B^{a c} F M O P Q U$ ed. | 4. alas] alis DFGIJ ed. | 5. recrescant] requiescant $A B^{a c}$ : quiescant $M U:$ crescant $F:$ concrescant $H \mid$ 6 . volet et videat] volat et videat $A B M U$ : volat et videt $B^{2} C F H O$ : volent et videant $J \mid 7$. et ipsi] ipsi $J$ : et ante $U$ :om. $M$ 


\section{Problèmes et perspectives sur la source «Physiologus » dans le DPR}

Dans l'ensemble des sources de philosophie naturelle qui nourrissent le DPR, dont le noyau fixe est constitué de citations d'Aristote et de ses commentateurs arabes et latins, d'Isidore et - seulement dans certaines sections - de Pline l'Ancien ${ }^{12}$, les notions liées au Physiologus occupent évidemment une place marginale et leur incidence est faible, tant dans l'économie globale de l'ouvrage de Barthélemy que dans celle des livres XII et XVIII et des chapitres particuliers où elles se trouvent. Leur nombre est restreint et leur extension est limitée, car elles ne dépassent pas en général la dizaine de lignes (sauf à propos de l'éléphant et du buffle), alors que la plupart des chapitres qui les contiennent ont une longueur remarquable et que le livre XVIII est l'un des plus longs de toute l'encyclopédie. Les citations peuvent porter sur un seul sujet ou constituer une petite collection de notices brèves : le premier cas s'illustre par exemple dans les chapitres De angue (sur le renouvellement de la peau du serpent), De panthera (sur l'attraction qu'elle exerce envers les autres animaux), De syrena (sur son danger pour l'homme); le second cas s'observe bien dans les chapitres De cocodrillo, De elephantibus, De lupo. Le choix du type d'information, comme son étendue, pourraient être conditionnés par la nature du modèle, car le Physiologus offre un éventail limité d'éléments, présentés presqu'exclusivement selon une interprétation éthique et allégorique, inspirée par une description rapide; de son côté, Barthélemy semble privilégier les descriptions « concrètes " portant sur les créatures les plus communes, en incluant les merveilleuses ${ }^{13}$.

Quelques problèmes émergent d'une première évaluation de ces passages. Des citations plus ou moins amples du DPR et toute celle du chapitre De taxo, bien qu'elles soient attribuées explicitement au Physiologus, ne se trouvent ni dans ses versions anciennes, ni dans les bestiaires qui en sont issus et qui ajoutent des matériaux provenant des Etymologiae d'Isidore de Séville. De manière générale, les formulations utilisées par Barthélemy s'éloignent souvent de celles propres aux différentes formes du Physiologus, ce qui suggère que l'encyclopédiste a réécrit ses citations, comme il est de coutume chez lui, mais aussi qu'il a pu tirer ses informations d'une source intermédiaire indéterminée. D'ailleurs, les six passages finaux ont eux aussi affaire avec le Physiologus, mais leur attribution à cette source est problématique.

En effet, les citations de XVIII 14 De fenice, 22 De kaladrio, 29 De pellicano ont un marqueur de source que l'on pourrait définir comme « controversé » : rares sont les témoins qui portent ici le marqueur Physiologus, alors que les autres évoquent le Philosophus (la source philosophique par excellence, Aristote) tout comme l'édition de référence de 1601 ; dans le cas du pélican, l'édition de 1601 et le manuscrit $R$ portent le marqueur Plinius. Étant donné la qualité des manuscrits qui ont le marqueur correct, nous faisons l'hypothèse que l'utilisation de Philosophus et Plinius soit due à une confusion d'une partie de la tradition manuscrite : un échange se serait produit entre les noms de ces auctoritates, peut-être facilité par leur ressemblance au niveau paléographique, surtout s'ils étaient écrits sous forme abrégée.

Quant aux trois extraits provenant de XII 1 De aquila, XII 37 De upupa, XVIII 14 De bubalo, le premier est accompagné par le marqueur Plinius; le deuxième est introduit par le marqueur physici et se termine avec celui d'Isidore; le troisième se trouve après un 
long passage attribué à la Naturalis historia et, bien qu'il soit entièrement tiré du Physiologus, cette source n'est mentionnée qu'à la fin du passage. La même confusion paléographique évoquée pour les trois cas précédents peut justifier la présence de Plinius dans le passage sur l'aigle; dans le cas du chapitre De bubalo, il est aussi plausible que le marqueur Physiologus se soit déplacé pendant le processus de copie à la fin de la citation, ou qu'il ait disparu de son début. Une explication paléographique pourrait être proposée également pour le marqueur physici du chapitre De upupa, assez proche de Philosophus et Physiologus; quant à la fausse attribution à Isidore de ce passage, qui ne se trouve pas dans les Etymologiae, elle pourrait dériver d'une confusion due à la similarité des contenus des deux ouvrages. Dans ces dernières citations, cependant, l'erreur est répandue dans tous les manuscrits collationnés, ce qui suggère qu'il s'agit d'une confusion propre à une partie considérable et peut-être même à toute la tradition du $D P R$. Le responsable pourrait alors être l'auteur lui-même, qui aurait fait une confusion dans les marqueurs de source au moment d'en recopier les extraits ou de les réorganiser dans sa copie de travail. Sinon, le DPR aurait hérité ces erreurs d'une source qui n'était pas une version du Physiologus, mais un ouvrage qui en reprenait des extraits, en les reformulant et en les mélangeant peut-être avec d'autres textes. Cela nous reconduit à la question fondamentale qui nous occupe, à savoir si Barthélemy a vraiment exploité une version du Physiologus, ou s'il a plutôt connu ce texte à travers un intermédiaire.

\subsection{Le DPR et les bestiaires issus du Physiologus}

Pour ne rappeler ici que les grands axes d'évolution du texte connu comme Physiologus latinus ${ }^{14}$, quatre versions anciennes sont d'abord à prendre en considération : il s'agit de $A, B, C^{15}$ et $Y^{16}$ (selon les sigles adoptés par leurs premiers éditeurs et encore de référence). Les deux dernières sont les plus proches du texte grec, mais s'apparentent à deux familles différentes et $C$ est aussi proche de la version éthiopienne du Physiologus ; $A$ emprunte des chapitres tantôt à $Y$ et tantôt à $B$, dont l'origine n'est pas résolue. La version $B$ forme à son tour le noyau de toutes les familles de bestiaires qui se diffusent à partir du XII ${ }^{e}$ siècle et qui ajoutent au texte de $B$ des passages tirés des Etymologiae d'Isidore de Séville tout en réorganisant les chapitres et leur contenu d'une façon de plus en plus radicale. La première de ces versions, dont les autres découlent, est celle connue comme B-Is (B-Isidorus), qui introduit les apports isidoriens généralement en fin de chapitre ${ }^{17}$. De B-Is descend le remaniement $H-B-I s^{18}$, qui fut la source principale de $S$ ( Bestiaire de seconde famille») ${ }^{19}$, réorganisant et amplifiant énormément, sur la base d'Isidore, la structure originaire de B-Is. Un autre bestiaire, connu sous le sigle de Tra (Bestiaire "Transitional »), naît à l'époque en tirant ses contenus de B-Is, H-B-Is et $S^{20}$. Une autre version encore est $H$, dont le modèle direct a été identifié par R. Baxter comme étant la version H-B-Is ; cependant, l'étude conduite par E. Kuhry sur certains passages de $H$ semble suggérer que son texte dérive plutôt d'un double modèle, à savoir $B$-Is et $H-B-I s^{21}$. Enfin, nous ne traiterons ni du bestiaire $T$ ("Troisième famille »), plus tardif, ni d'une forme du Physiologus indépendante de B-Is comme les Dicta Chrysostomi, ni de deux versions poétiques telles que le Physiologus Theobaldi (produit à la fin du $\mathrm{XI}^{\mathrm{e}}$ siècle et très diffusé) et le Bestiaire de Philippe de Thaon (dont le modèle serait encore de type B-Is).

Que le DPR s'apparente à la version $B$, et plus proprement à B-Is, est évident : tant au niveau des contenus que de la formulation des notices, le DPR s'éloigne de l'autre 
version principale du Physiologus, c'est-à-dire $Y$; il transmet d'ailleurs, mélangées au texte du Physiologus, des informations isidoriennes qui se trouvent déjà dans B-Is. Voici quelques exemples :

\begin{tabular}{|c|c|c|}
\hline $\begin{array}{l}\text { De onagro. XXV die Martii duodecies in die et } \\
\text { totiens in nocte rugit, per cuius rugitum } \\
\text { equinoctium apud Affros discernitur, et dicit } \\
\text { quod totiens semper rugit de die quot dies habet } \\
\text { horas, similiter et de nocte. Unde et sylvani in } \\
\text { montibus Affrice, in quibus onagri abundant, } \\
\text { per numerum rugitus eorum numerum } \\
\text { horarum, dierum et noctium computant et } \\
\text { diversificant. }\end{array}$ & $\begin{array}{l}\text { B/B-Is XXI Vicesimo quinto } \\
\text { die mensis Famenoth, qui est } \\
\text { Martius, duodecies in nocte } \\
\text { rugit, similiter et in die; et } \\
\text { ex hoc cognoscitur quia } \\
\text { equinoctium est diei vel } \\
\text { noctis, et numerum } \\
\text { horarum a rugitus onagri } \\
\text { per singulas cognoscunt } \\
\text { horas, semel rugientis. }\end{array}$ & $\begin{array}{l}\text { Y XXV In quinta et vigesima } \\
\text { Famenoth mensis } \\
\text { cognoscunt ab onagro } \\
\text { quoniam equitas dierum fit: } \\
\text { si autem clamaverit } \\
\text { duodecies, cognoscit rex et } \\
\text { palatium quoniam equitas } \\
\text { dieifiet. }\end{array}$ \\
\hline $\begin{array}{l}\text { De bubalo }{ }^{22} \text {. Caput submittit inter frutices } \\
\text { subtilia et prolixa virgulta sive vimina habentes } \\
\text { [...] cumque diu luctans ab illis ligaminibus se } \\
\text { non expedit, immo fortius se involvit, pre } \\
\text { indignatione alte mugit. }\end{array}$ & 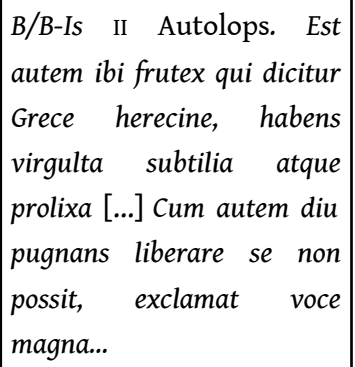 & $\begin{array}{l}\text { Y II De autolope. Sunt } \\
\text { autem ibi ricine (dicuntur } \\
\text { Grece), hoc est frutices } \\
\text { tenues ramos habentes [...] } \\
\text { Et clamat, volens fugere, et } \\
\text { non potest, obligatus est } \\
\text { enim... }\end{array}$ \\
\hline \multicolumn{2}{|c|}{$\begin{array}{l}\text { De onocentauro. Onocentaurum esse compositum ex humana effigie et } \\
\text { asinina. Nam ab umbilico et sursum figuram habet hominis et ab eodem } \\
\text { inferius formam obtinet animalis. }\end{array}$} & $\begin{array}{l}\text { B XII Superior pars homini } \\
\text { similis est, inferioris vero } \\
\text { partis membra sunt nature } \\
\text { valde agrestis. } \\
\text { add. B-Is Onocentaurus } \\
\text { autem vocatur eo quod } \\
\text { media specie sit homo, media } \\
\text { vero asinus. }\end{array}$ \\
\hline \multicolumn{2}{|c|}{$\begin{array}{l}\text { De cocodrillo }{ }^{23} \text {. Cocodrillus, si quendam invenit hominem iuxta littus, } \\
\text { interficit eum si potest, et postea plorat super eum et postea devorat ipsum. Et } \\
\text { dicit quod de eius fimo fit unguentum, unde facies mulierum sophisticantur, } \\
\text { ut senes et rugose iuvencule per tempus videantur. Herbas bonas libenter } \\
\text { comedit, inter quas enidros serpens parvulus, qui ei inimicatur, caute se } \\
\text { involvit [...] et sic interimit eum et occidit et sic illesus exit. }\end{array}$} & $\begin{array}{l}\text { add. B-Is (à la fin du } \\
\text { chapitre consacré à } \\
\text { l'hydrus) } \\
\text { Hic, dum invenit hominem, } \\
\text { si poterit eum vincere } \\
\text { comedit, et semper plorat } \\
\text { illum. [...] De stercore eius } \\
\text { unguentum fiebat, unde } \\
\text { vetule et rugose meretrices } \\
\text { faciem suam perungebant, et } \\
\text { ruge extendebant fiebantque } \\
\text { pulcre [...] Hunc hydrus } \\
\text { deglutitus et unguibus et } \\
\text { dentibus interimit et vivus } \\
\text { inde exiit. }\end{array}$ \\
\hline
\end{tabular}


Barthélemy semble cependant lire aussi, dans sa version du Physiologus, d'autres informations provenant des Etymologiae, ce qui conduit à une comparaison entre les principales formes de bestiaire issues de B-Is pour déterminer si l'une est plus proche du DPR que les autres. Un petit indice en ce sens vient déjà de l'extrait sur le crocodile que nous avons cité, en particulier de deux détails, à savoir que l'animal pleure l'homme après l'avoir tué et que l'hydrus sort indemne de son ventre. Ces notices ne sont pas distribuées uniformément dans $B$-Is et dans ses descendants : la première, propre à $B-I S, H$ et $T$, manque dans $H-B-I s$ et $S$; la seconde se trouve sous la forme vivus exit dans B-Is et sous la forme illesus exit du DPR dans H-B-Is, $H$ et $S$ ( $T$ a les deux, à deux endroits différents):

\begin{tabular}{|c|c|c|c|}
\hline $\begin{array}{l}\text { H-B-Is non solum vivus, } \\
\text { sed etiam exit illesus }\end{array}$ & H non solum vivus, sed etiam illesus & & T exit vivus / exit illesus \\
\hline $\begin{array}{l}\text { Quod si aliquando } \\
\text { invenerit } \\
\text { comedit eum } \\
\text { potest. }\end{array}$ & $\begin{array}{l}\text { Quod si aliquando invenerit } \\
\text { hominem, comedit eum si vincere } \\
\text { potest, et postea eum semper plorat. }\end{array}$ & illesus & $\begin{array}{l}\text { Hic, dum invenerit hominem, si } \\
\text { poterit eum vincere comedit, } \\
\text { post et semper plorat eum. }\end{array}$ \\
\hline
\end{tabular}

Par ailleurs, aucun chapitre sur le loup ne se trouve dans les versions anciennes du Physiologus et dans B-Is : il ne fait son apparition que dans les bestiaires $H$-B-Is, $H, S$ et $T$, contenant tous la plus grande partie des informations données par le DPR selon une formulation assez constante.

Parmi ces versions, $S$ et $T$ s'alignent sur les expressions de B/B-Is et du DPR pour le chapitre sur l'autolops, alors que le bestiaire $H$ utilise des formulations différentes (Sunt autem ibidem virge urtice virides et molles; vociferatur [...] rugitu) et H-B-Is n'a aucune information. Dans le passage sur la panthère, les versions les plus proches du DPR sont $H-B-I s, S, T$ et plus éloignées sont $B / B-I s$ et $H$ :

\begin{tabular}{|l|l|l|l|}
\hline De panthera. Cum autem & H-B-Is (= S T) Cum ergo & B/B-Is XXIII Cum ergo comederit et \\
comederit et saturatus fuerit, & comederit et satiatum fuerit, & satiaverit se diversis venationibus, \\
se recondit in spelunca et & recondit se in spelunca sua et & recondit se in speluncam suam, ponit se et \\
dormit [...] Solus autem draco, & dormit [...] Solus autem draco, & dormit [...] Solus autem draco, cum \\
audiens vocem eius, timore & audiens eius vocem, timore & audierit vocem eius, timore contrahitur et \\
perterritus fugit in cavernam, & perterritus fugit in cavernis & fulcit se in terraneis cavernis terre, ibique \\
nec ferens odorem in semetipso & terre; ibi non ferens odorem & non ferens vim suavitatis odoris in \\
deficit et torpescit. & torpescit in semetipso. & semetipsum contractus obtorpescit ${ }^{24}$. \\
\hline
\end{tabular}

Encore, le chapitre sur le serpent ne semble se trouver, au sein de la tradition découlée de $B$, que dans les bestiaires $S$ et $T$, alors qu'il était présent dans la version $Y$ et en conséquence dans $A$ : 
De angue. Anguis se sentiens morbo vel senio aggravatum pluribus diebus abstinet a cibo et ieiunat, ut sic pellis eius a carne facilius relaxetur [...] et sic angustam rimam petre alicuius seu cavernam querens per rime angustiam intrat et, cum quadam violentia transiens, ab exuvia penitus se decorticat ac denudat.
$S / T$ De serpente. Cum senuerit caligant oculi eius, et si voluerit novus fieri abstinet se et ieiunat multis diebus, donec pellis eius relaxetur; et querit angustam rimam in petra, et intrat in eam et contribulat se et deponit veterem pellem.

Dans le cas du De elephantibus, en revanche, seul $S$ se rapproche du DPR, bien que ce dernier introduise une variation importante :

De elephantibus. Cadit subito et succumbit. Qui casum suum S Cadens autem clamat fortiter [...] et videns irrecuperabilem miro modo barrit, id est clamat et rugit; veniunt duodecim elephantes et non ad cuius barritum multitudo iuvenum subito accedit possunt eum levare qui cecidit. Deinde elephantum, qui paulatim seniorem pro viribus elevant et, ut clamant omnes et statim venit pusillus eum relevent, miro affectu se totis viribus inclinant.

elephans [...] et elevat eum.

Quant au passage sur la sirène, déjà étudié par E. Kuhry, on se limite à souligner que le DPR offre deux précisions qui ne se trouveraient ensemble que dans H-B-Is (et parfois dans $T)$ :

\begin{tabular}{|l|l|l|}
\hline $\begin{array}{l}\text { De sirena. Ab umbilico et } \\
\text { sursum habens formam } \\
\text { virginis, inferius figuram } \\
\text { piscis. }\end{array}$ & $\begin{array}{l}\text { B/B-IS XII (= S) Usque ad umbilicum } \\
\text { figuram hominis habent, extrema vero } \\
\text { Hars usque ad pedes volatilis habent } \\
\text { feminas, ab umbilico vero et infra } \\
\text { piscisfiguram habentes. }\end{array}$ & $\begin{array}{l}\text { figuram. } \\
\text { Usque ad umbilicum figuram hominis } \\
\text { habent, extrema vero pars usque ad } \\
\text { pedes volatilis vel piscis habet figuram }\end{array}$ \\
\hline
\end{tabular}

Il semble en conclusion que Barthélemy ait connu le texte du Physiologus dans une version issue de $B-I s$, proche pour certains aspects de $H$-B-Is, pour d'autres plutôt de $S$, qui découle de H-B-Is après une série d'ajouts et de remaniements. Il est donc impossible, du moins sur la base des témoins que nous avons consultés, d'identifier une seule version comme suffisante pour justifier l'ensemble des informations que le DPR reprend du Physiologus.

\subsection{Le $D P R$ et de possibles sources intermédiaires}

En même temps, le DPR s'éloigne de toutes les versions connues du Physiologus en raison de reformulations occasionnelles de leur texte et de certains contenus en plus. Comme on l'a dit, toute la citation du chapitre De taxo est attribuée erronément au Physiologus, et d'autres informations absentes de la même source se trouvent dans la seconde partie du chapitre De onagro (qui raconte comment l'âne sauvage séduit sa femelle) et à la fin des chapitres De elephantibus (qui dit de quelle manière deux vierges peuvent chasser l'éléphant) et De syrena (qui raconte comment elle enlève et tue les marins). Il s'impose à ce point de vérifier si ces passages ne viennent pas d'un texte qui utilisait une version 
récente du Physiologus, sélectionnée, reformulée et mélangée avec des notices tirées d'autres sources. Si un texte de ce type existe, il pourrait également être la source d'autres ou de tous les extraits attribués au Physiologus que nous avons étudiés jusqu'ici.

Le texte qui pourrait mieux se prêter à jouer ce rôle est une compilation anonyme de proprietates rerum témoignée par une dizaine de codices, en deux versions longues et une abrégée, dont la forme originaire, perdue, pourrait avoir été composée entre 1220 et 1225/1226 selon les études de J. Deus ${ }^{26}$. Cette forme correspondrait au texte que Thomas de Cantimpré appelle dans le prologue à son Liber de natura rerum l'Experimentator, et sa première version (Fassung I) aurait été une source du DPR. Si H. Meyer et B. Van den Abeele ont soutenu que ce texte aurait plutôt utilisé le DPR et le vrai Experimentator, à savoir une autre compilation qui serait perdue, des recherches récentes semblent infirmer cette hypothèse et soutenir celle de J. Deus, car le DPR a introduit dans sa forme originale des passages du texte qu'elle édite ${ }^{27}$. À la lumière de ces circonstances, nous avons vérifié que la plus grande partie des animaux dont il est question dans notre enquête se trouve aussi dans l'Experimentator, et que certaines créatures sont traitées de façon identique dans le Physiologus et dans l'Experimentator, comme l'onocentaure :

Fass. I XIII 47.1, p. 236 (cf. Kurzfass. VIII 38, p. 351) Onocentaurus ex duabus naturis constat, ut dicit Phisiologus. Superior pars assimilatur homini, inferior asino.

Plus fréquemment, cependant, nos animaux sont présentés par l'Experimentator d'une façon différente au niveau des contenus et de la formulation, alors que le Physiologus et le DPR restent proches l'un de l'autre. Dans le cas du De angue, le texte de l'Experimentator ressemble à celui du Physiologus (chapitre De serpente) et du DPR, mais par rapport à ces deux textes il omet l'expression abstinet se/a cibo et le terme angustam rimam est substitué par angustum foramen:

Fass. I XIII 90.1, p. 272 (cf. Kurzfass. VIII 47, p. 353) Cum senuerit, caligant oculi eius, et si senex voluerit novus fieri, ieiunat multis diebus, donec pellis eius relaxetur, et querit angustum foramen in petra et in eum intrat et contribulat se et deponit pellem veterem.

Pour le chapitre De cocodrillo, l'information sur l'onguent issu de cet animal manque dans l'Experimentator; l'épisode de l'hydrus est effectivement introduit d'une façon plus proche du DPR que dans le Physiologus (on décrit le serpent s'enroulant dans les herbes que le crocodile mange, alors que dans le Physiologus il attaque plutôt le crocodile dormant à la manière de la mangouste-ichneumon), mais l'expression finale de l'Experimentator s'éloigne de l'illesus exit du DPR, de H-B-Is et de ses avatars :

Fass. I XIII 67.8 et 5, p. 261-262 (cf. Kurzfass. VIII 51, p. 353) Qui, si aliquando invenerit hominem, commedit illum, si vincere potest, et super eum plorat; Herbas bonas commedit [...] Unde quidam serpens naturaliter inter herbas se involvit [...] qui in interiora corporis foramen facit et sic interficiendo ipsum exit.

Quant aux informations contenues dans le De onagro et le De panthera, elles sont aussi dans l'Experimentator, mais exprimées d'une façon divergente du Physiologus et du DPR:

Fass. I XIII 46.4 et 7, p. 236 (cf. Kurzfass. VIII 37, p. 351) Horas diei et noctis naturaliter cognoscunt, unde duodecies in die et duodecies in nocte rugiunt [...] Animal est inpatiens fervens libidine, unde ascendit in montem contra ventum et delectatur in illo vento ${ }^{28}$.

Fass. I XIII 49.2 et 4, p. 240 (cf. Kurfass. VIII 40, p. 351-352) Mansuetum est nimis, in spelunca se abscondit, tribus diebus dormit, tertia die surgit, mire suavitatis odorem effundit et rugitum emittit. Ad eius rugitum animalia gressus figunt et pantheram precedentem ordinate sequuntur [...] Solus draco eius vocem audiens fugit in speluncam et dormit et quasi victus aures obturescit et obmutescit. 
Les notices que le De elephantibus ajoute par rapport au Physiologus sont également absentes de l'Experimentator, et d'autres propres au Physiologus et au DPR sont omises ou formulées d'une façon légèrement différente, comme il arrive dans le De lupo. Notons cependant que l'Experimentator fait allusion, comme le DPR, à la force qui réside dans les ongles du loup, force que le Physiologus met en relation avec la poitrine et la bouche et Isidore avec les pattes.

Fass. I XIII 18.1, 2, 8, 10, p. 213-215 (cf. Kurzfass. VIII 16, p. 349) Intellectu et memoria multa vigent [...] Sedet et flectit pedes suos, set non potest flectere quatuor pedes simul [...] dormit stante corpore [...] Si elefans voluerit facere filios, vadit ad orientem [...] si ceciderit, non potest surgere...

Fass. I XIII 34.1 et 8, p. 228-229 (cf. Kurzfass. VIII 29, p. 350) In unguibus et pedibus sit illi virtus [...] pilum habet in cauda villosa [...] qui excitat ad amorem [...] Oculos habet igneos et quasi lux emicantes [...] Collum non flectit et retro est debilis ${ }^{29}$.

Enfin, l'Experimentator ne comporte aucun passage consacré au buffle, à la sirène ou au blaireau ; il y a juste une allusion, dans le chapitre sur le renard, au fait qu'il usurpe la maison de ce dernier (Fass. I XIII 60.2, p. 249 Usurpat sibi alienam domum et ingreditur etiam taxi domum).

La situation est différente pour les extraits sur les oiseaux, car l'Experimentator offre, dans des passages explicitement attribués au Physiologus (sauf les deux derniers, mis en relation avec Augustin et les philosophi), des expressions plus proches du DPR que ce qu'on lit dans B/B-Is et dans tous les bestiaires. Les expressions sont soulignées cidessous :

\begin{tabular}{|c|c|c|}
\hline $\begin{array}{l}\text { De fenice. De hac ave dicit } \\
\text { Physiologus [...] vivens cCC vel D } \\
\text { annis; quibus completis, cum suum } \\
\text { sentit defectum, nidum facit ex lignis } \\
\text { aromaticis et multum siccis, qui in } \\
\text { estate ex fervore solis flante Favonio } \\
\text { accenduntur [...] infra triduum } \\
\text { quidam vermiculus nascitur, qui } \\
\text { paulatim plumas recipiens in } \\
\text { volucrem reformatur. }\end{array}$ & $\begin{array}{l}\text { Fass. I IX 16.4, p. } 158-159 \text { (cf. } \\
\text { Kurzfass. IV 36, p. } 316 \text { ) Ut enim ait } \\
\text { Phys<i>ologus, expletis quingentis } \\
\text { annis cum suum sentiat defectum in } \\
\text { lignis Lybani nidum facit et diversa } \\
\text { colligens aromata [...] et flante } \\
\text { Favonio [...] acervum succendit [...] } \\
\text { prima die vertitur in vermem, } \\
\text { secunda in volucrem, post hec in } \\
\text { pristinum revocatur statum. }\end{array}$ & $\begin{array}{l}\text { B/B-Is IX Expletis quingentis } \\
\text { annis vite sue, intrat in lignis } \\
\text { Libani et replet utrasque alas } \\
\text { diversis aromatibus [...] } \\
\text { ignem ipse sibi incendit [...] } \\
\text { ibi vermiculum modicum } \\
\text { suavissimo odore fragrantem } \\
{[. . .] \text { tertia die [...] statu suo }} \\
\text { integram atque perfectam } \\
\text { avem phenicem. } \\
\text { H-B-Is (= S T) Quingentos } \\
\text { annos vivere fertur et ultra; } \\
\text { dum se viderit senuisse } \\
\text { collectis (aromatum add. S) } \\
\text { virgultis rogum sibi instruit } \\
{[. . .] \text { Postea vero tertia die }} \\
\text { nova avis de cineribus } \\
\text { surgit }{ }^{30} \text {. }\end{array}$ \\
\hline
\end{tabular}




\begin{tabular}{|c|c|c|}
\hline $\begin{array}{l}\text { De kaladrio. Kaladrius secundum } \\
\text { Physiologum est albi coloris, nullam } \\
\text { habens partem nigredinis, cuius pars } \\
\text { inferior femoris ipsius purgat } \\
\text { caliginem oculorum. }\end{array}$ & $\begin{array}{l}\text { Fass. I IX 23.1, p. } 165 \text { Caladrius } \\
\text { secundum Phisiologum est albi } \\
\text { coloris, nullam habens partem } \\
\text { nigredinis. Cuius pars inferior ipsius } \\
\text { femoris caliginem purgat oculorum. }\end{array}$ & 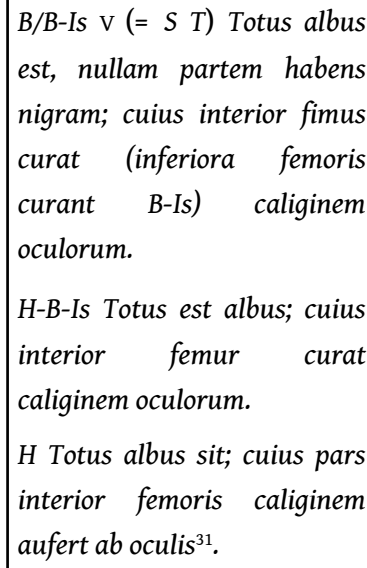 \\
\hline $\begin{array}{l}\text { De pellicano. Et idem dicit } \\
\text { Physiologus [...] parentes suos in } \\
\text { faciem percutiunt, propter quod ipsos } \\
\text { mater repercutit et occidit. }\end{array}$ & $\begin{array}{l}\text { Fass. I IX 30.4, p. } 172 \text { (cf. Kurzfass. } \\
\text { IV } 50 \text { p. 318) Phisiologus dicit [...] } \\
\text { parentes suos in faciem percutiunt, } \\
\text { qua de re ipsos mater repercutit et } \\
\text { interficit. }\end{array}$ & $\begin{array}{l}\text { B/B-IS VI }\left(=\begin{array}{llll}H-B-I S & H & S & T\end{array}\right) \\
\text { Percutiunt parentes suos in } \\
\text { faciem; parentes autem eorum } \\
\text { repercutiunt eos et occidunt. }\end{array}$ \\
\hline $\begin{array}{l}\text { De aquila. Ad hoc dicit Augustinus } \\
\text { et Plinius [...] in senectute patitur } \\
\text { caliginem in oculis et gravedinem in } \\
\text { alis suis, contra quod incommodum } \\
\text { instruitur a natura ut fontem aque } \\
\text { scaturientis querat. Deinde ascendit } \\
\text { quantum potest per aera, donec ex } \\
\text { calore aeris et labore volatus fortius } \\
\text { incalescat [...] subito descendens in } \\
\text { fontem ruit; ibi mutatis plumis et } \\
\text { purgata caligine in oculis... }\end{array}$ & $\begin{array}{l}\text { Fass. I IX 2.9, p. } 138 \text { (cf. Kurzfass. IV } \\
\text { 20, p. 314) Dicit insuper Augustinus } \\
\text { [...] quando in alis gravedinem et in } \\
\text { oculis caliginem [...] senserit [...] } \\
\text { fontem aque scaturientis querit [...] } \\
\text { ex motu et aeris calefactione et solis } \\
\text { appropinquatione se calefaciens } \\
\text { subito in fontem descendit, immutatis } \\
\text { plumis et purgata oculorum caligine... }\end{array}$ & $\begin{array}{l}\text { B/B-Is VIII }(=S T) \text { Cum } \\
\text { senuerit, gravantur ale eius, } \\
\text { et obducuntur oculi eius } \\
\text { caligine; tunc querit fontem } \\
\text { aque, et contra eum fontem } \\
\text { evolat in altum usque ad } \\
\text { aerem solis, et ibi incendit } \\
\text { alas suas [...] tunc demum } \\
\text { descendens ad fontem trina } \\
\text { vice se mergit, et statim } \\
\text { renovatur tota... }\end{array}$ \\
\hline $\begin{array}{l}\text { De upupa. De hac dicunt physici } \\
\text { quod, cum senuerit ita quod nec } \\
\text { videre nec volare queat, pulli eius } \\
\text { evellunt ei pennas invalidas [...] } \\
\text { donec recrescant plume eius et sic } \\
\underline{\text { renovata perfecte volet et videat }} \\
\text { clare sicut et ipsi. }\end{array}$ & $\begin{array}{l}\text { Fass. I IX 39.3, p. } 182 \text { (cf. Kurzfass. } \\
\text { IV 60, p. 320) De hac dicunt } \\
\text { phylosophi quod cum senuerit ita, } \\
\text { quod volare vel videre nequeat, pulli } \\
\text { eius evellunt ei invalidas pennas [...] } \\
\text { donec recrescant penne eius et } \\
\frac{\text { renovata sit et videat clare sicut }}{\text { ipsi }^{33} \text {. }}\end{array}$ & $\begin{array}{l}\text { B/B-Is X (= T) Cum viderint } \\
\text { parentes suos senuisse, et } \\
\text { neque volare posse, neque } \\
\text { videre, pre caligine oculorum, } \\
\text { tunc filii eorum evellunt } \\
\text { vetustissimas pennas } \\
\text { parentum suorum [...] donec } \\
\text { recrescant penne eorum et } \\
\text { reilluminentur oculi eorum, } \\
\text { ita ut totum corpore renovari } \\
\text { possint sicut antea, et videre } \\
\text { et volare }{ }^{34} \text {. }\end{array}$ \\
\hline
\end{tabular}

Il est possible que Barthélemy ait décidé de se servir d'une source qu'il exploitait déjà pour d'autres raisons afin d'intégrer les descriptions des oiseaux sans remonter au texte d'origine. L'emprunt aurait été parfois stimulé par la présence dans l'Experimentator de la mention explicite du Physiologus, ce qui permet de revenir à l'analyse des marqueurs de source conduite plus haut, pour confirmer que dans les chapitres De fenice, De kaladrio et De pellicano il faut retenir cette attribution au lieu de 
Philosophus ${ }^{35}$. L'attribution incorrecte du De aquila et De upupa (à Plinius, physici, Isidorus) serait en revanche de Barthélemy ou de la tradition du DPR, car elle ne se trouve pas dans la source intermédiaire utilisée, à savoir l'Experimentator, au moins selon le texte édité par J. Deus.

Pour essayer de retracer les informations du DPR sans parallèle dans les versions du Physiologus, nous avons fait aussi quelques sondages sur les "recueils de propriétés » qui se diffusent peu avant les encyclopédies du plein XIII siècle : bien qu'ils aient été primitivement conçus comme outils de travail pour la prédication, ces recueils ont été aussi utilisés par les compilateurs de philosophie naturelle, car ils conjuguent le savoir sur la nature et les apports du Physiologus latin et des bestiaires du XII ${ }^{\mathrm{e}}$ siècle. Grâce aux récents travaux d'E. Kuhry, les relations entre les recueils les plus importants de l'époque se clarifient progressivement ${ }^{36}$, et sur la base de ses considérations j'ai donc consulté les manuscrits suivants: Troyes, Bibliothèque municipale, 1236, un dictionnaire encyclopédique du deuxième tiers du XIII siècle connu comme l'Anonyme de Clairvaux; Montpellier, Bibliothèque internuniversitaire de Médecine, $\mathrm{H}$ 470, qui est l'un des deux témoins du recueil nommé Abies arbor alta d'après son incipit (l'autre, Bruxelles, Bibliothèque royale, II 1060, remonte également à la première moitié du XIII ${ }^{e}$ siècle et présente un état un peu différent du texte); Paris, Bibliothèque nationale de France, lat. 11207, lat. 14297 et lat. 16079 du célèbre recueil dit Angelus purus natura, largement diffusé au XIII ${ }^{\mathrm{e}}$ siècle et qui pourrait être la source des deux compilations précédentes ${ }^{37}$. Il en ressort que de rares notices et animaux traités dans le DPR et le Physiologus se trouvent aussi dans ces compilations, qui ne comprennent aucune des informations ajoutées par Barthélemy; de plus, leurs descriptions zoologiques sont rapides ou remaniées à un tel point qu'il est impossible que le DPR les ait utilisées à la place du Physiologus.

Une comparaison du DPR avec d'autres encyclopédies contemporaines, telles que le Liber de natura rerum de Thomas de Cantimpré ${ }^{38}$ et le Speculum naturale de Vincent de Beauvais ${ }^{39}$, fait en revanche émerger des éléments intéressants, bien que non décisifs. Le Liber contient à peu près les mêmes informations que celles que Barthélemy donne à propos du blaireau, mais réparties dans deux chapitres : l'un est consacré à l'hemtra, et les notices sont attribuées au mystérieux Liber rerum ${ }^{40}$; l'autre porte sur le renard, et l'information est attribuée à l'Experimentator.

LDNR IV 38.1. Hemtra, ut dicit Liber rerum, in partibus Germanie animal modicum admodum est. Mas vero et femina in estate cybos congerunt, quibus vivant in hyemem [...] Naturaliter autem femina in sumendis cibis avida est et prodiga. Econtrario masculus parcus et avarus super modum est adeo, ut vix sibi vel tenuissime vite alimoniam velit impendere. Masculus autem aviditatem femine sciens aditum obstruit, per quem cibos absconditos possit attingere. Femina autem naturaliter callida est: avida autem sibi foramen facit occultum ex altera parte aditus oppositi, per quod possit furari mare nescio sufficientiam sue aviditati...

LDNR IV 109.5. Dicit Experimentator quod vulpes in fodiendis foveis non laborat, sed bestia quedam, quam daxum dicimus; daxus enim cum foveam in terram foderit ad quietem, vulpes dolosa in ingressu fovee ventrem suum stercoribus exonerat, ut fetore digestionis putride fovea polluta vilescat; et revera daxus bestia fetorem digesti stercoris abhominabiliter detestatur locumque dimittit; et sic dolosa bestia locum possidet.

Vincent de Beauvais connaît la seconde information, mais lui attribue une source différente (Pline), alors que sa description du creusement de la tanière du blaireau, attribuée au Physiologus ${ }^{41}$, vient d'Alexandre Neckam, De naturis rerum, ch. 127 De taxo et vulpe $e^{42}$. 
Par ailleurs, le Liber de natura rerum ne donne qu'une source vague pour une notice sur la chasse à l'éléphant qui est très proche de la description donnée par le DPR :

LDNR IV 33.8. Et hec venatio elephantum. Narrat scriptura libri que continet veterum relationes quod elephas hoc modo capitur: Due puelle virgines nude in desertum pergunt, ubi habitant elephantes; una earum urnam, altera gladium ferens. Quibus alta voce cantantibus audit elephas, accurrit propere. Qui mox naturali instinctu virginee carnis innocentiam recognoscens, in eis amoris dulcedine castimoniam veneratur, lambensque earum pectus et ubera delectatus mirifice resolvitur in soporem. Nec mora puella cum gladio tenerum ventrem perfodiens elephantis sanguinem ruentis fundit, excipitque in urna altera puella fusum. Hoc sanguine regalis purpura tingitur.

Pour résumer, si les bestiaires que nous avons signalés plus haut, issus de B-Is, justifient un bon nombre de notices que le DPR attribue au Physiologus, d'autres informations particulières n'émergent ni de ceux-ci, ni de possibles sources intermédiaires que nous avons analysées, au moins selon les formes que nous en connaissons. Les passages du $D P R$ dont il est possible de retracer la provenance se révèlent un ensemble de matériaux tirés des bestiaires et d'extraits déjà filtrés par l'Experimentator, les uns et les autres retravaillés au niveau de la formulation d'une façon plus ou moins importante. Il reste possible que, comme pour les descriptions des oiseaux, les extraits sur les quadrupèdes découlent eux aussi d'une compilation intermédiaire, qui mélangeait l'apport de plusieurs bestiaires et d'autres sources de philosophie naturelle: ce pourrait être le cas d'une version amplifiée de l'Experimentator étudié par J. Deus, dont l'existence ne semble cependant pas attestée à l'heure actuelle.

\subsection{Le DPR et ses moralitates}

Il y a enfin un autre genre de rapprochement possible entre Physiologus et DPR, qui, même s'il ne révèle pas un rapport direct entre les deux textes, fait ressortir un ensemble de consonances et dissonances relatives à une thématique centrale du premier, qui prend dans le second une forme assez particulière. Si les inspirations symboliques à propos de chaque élément occupent la plus grande partie des chapitres du Physiologus, le texte principal du DPR ne réserve aucun espace à ces contenus. Cependant, les marges du DPR portent un grand nombre de notes moralisatrices, qui deviennent le seul moyen de véhiculer la signification exégétique d'un ouvrage essentiellement conçu pour la prédication. Bien que la critique tende à attribuer la conceptualisation de ces moralitates à l'auteur lui-même, le corpus de notes est extrêmement mouvant, car il varie d'un manuscrit (ou d'un groupe de manuscrits) à l'autre, tant par le nombre des notes que dans la formulation, et disparait peu à peu dans les témoins les plus tardifs, pour être ensuite complètement absent des éditions anciennes ${ }^{43}$. Or, il est évident que ces notes reflètent une culture commune aux clercs de la première moitié du XIII siècle et une série de topoi propres au milieu de la prédication de la fin du siècle précédent; de leur lecture se dégage par ailleurs l'impression que Barthélemy se serait fondé sur les caractéristiques les plus typiques de chaque animal et sur celles les plus aisément interprétables selon une vision chrétienne. Le but des tableaux qui suivent se limite dès lors à montrer que quelques descriptions tirées du Physiologus (ou de l'Experimentator) analysées plus haut sont accompagnées dans plusieurs témoins du DPR par des notes faisant écho aux interprétations symboliques de la source, bien qu'il soit difficile de supposer qu'elles en découlent d'une manière directe (comme il ne s'agit pas d'une analyse portant sur la 
littera du texte, nous nous contentons de reprendre l'allégorie dans la forme propre aux versions anciennes du Physiologus, B et $Y)^{44}$.

\begin{tabular}{|c|c|}
\hline $\begin{array}{l}\text { XII } 1 \text { De aquila } \\
\text { Nota remedium contra } \\
\text { peccatum } \\
\text { Nota de renovatione anime per } \\
\text { penitentiam vel baptismum }\end{array}$ & $\begin{array}{l}\text { B VIII (= Y VIII) Ergo et tu, homo, sive Iudeus sive gentilis, qui vestimentum } \\
\text { habes vetus, et caligantur oculi cordis tui, quere spiritalem fontem Domini. }\end{array}$ \\
\hline $\begin{array}{l}\text { XII } 22 \text { De kaladrio } \\
\text { Nota de innocentia Christi } \\
\text { Nota de gratie subtractione } \\
\text { Nota de respectu gratie divine }\end{array}$ & $\begin{array}{l}\text { B V }(=Y \text { V) Caladrius igitur personam accepit Salvatoris nostri: totus est } \\
\text { candidus [...] Veniens autem de excelsis celis suis ad infirmum populum } \\
\text { Iudeorum, avertit faciem suam ab eis propter incredulitatem } \\
\text { eorum; convertit se ad nos gentes, tollens infirmitates nostras. }\end{array}$ \\
\hline $\begin{array}{l}\text { XII } 29 \text { De pellicano } \\
\text { Nota de Christo sive de prelato } \\
\text { Nota de contemptu Dei } \\
\text { Nota de morte Christi } \\
\text { Nota de effectu passionis }\end{array}$ & $\begin{array}{l}\text { B VI (= Y VI) Ita et Dominus noster Iesus Christus per Isaiam prophetam } \\
\text { dicit: Filios genui et exaltavi, ipsi autem spreverunt me. Genuit igitur auctor } \\
{[\ldots . .] \text { nos vero e contrario percussimus eum in faciem. }}\end{array}$ \\
\hline $\begin{array}{l}\text { XVIII } 8 \text { De angue } \\
\text { Nota de abstinentia } \\
\text { Nota de amaritudine } \\
\text { contritionis et confessionis } \\
\text { Nota de fletu et devotione } \\
\text { Nota de angusta penitentia }\end{array}$ & $\begin{array}{l}\text { Y XIII Et nos, per multam abstinentiam et tribulationem, pro Christo } \\
\text { deponemus veterem hominem et indumentum eius. }\end{array}$ \\
\hline $\begin{array}{l}\text { XVIII } 14 \text { De bubalo } \\
\text { Nota contra gulam } \\
\text { Nota quod carnales deliciis et } \\
\text { divitiis sepe malo obvolvuntur } \\
\text { Nota quod vix recedit homo a } \\
\text { mala consuetudine } \\
\text { Nota quod demon multos } \\
\text { interficit in deliciis magnis } \\
\text { Nota quod demones multos } \\
\text { seducunt per minora vitia quos } \\
\text { non audent aggrediper maiora }\end{array}$ & $\begin{array}{l}\text { B II (= Y II) Sic et tu, homo Dei, qui studes sobrius esse et castus et } \\
\text { spiritaliter vivere }[. . .] \text { Cave ergo, homo Dei, ebrietatem, ne obligeris luxurie } \\
\text { et voluptati, et interficiaris a diabolo. }\end{array}$ \\
\hline $\begin{array}{l}\text { XVIII } 28 \text { De castore } \\
\text { Nota quod homo debet se } \\
\text { castrare propter Deum }\end{array}$ & $\begin{array}{l}\text { B XVII (= Y XXXVI) Sic et omnis qui secundum mandatum Dei conversatur, et } \\
\text { caste vult vivere, abscidit a se omnia vitia et omnis impudicitie actus, et } \\
\text { proiciat post se in faciem diaboli. }\end{array}$ \\
\hline $\begin{array}{l}\text { XVIII } 77 \text { De onocentauro } \\
\text { Nota de ypocritis qui in occulto } \\
\text { sunt carnales }\end{array}$ & B XII $(=Y \mathrm{XV})$ Huic assimilantur vecordes atque bilingues homines informes. \\
\hline
\end{tabular}


Quelques autres moralitates peuvent être comparées avec les contenus du Physiologus, à savoir les notes qui se réfèrent à des animaux présents dans cette source et que Barthélemy décrit sur la base d'autres ouvrages, en les interprétant toutefois d'une manière semblable.

\begin{tabular}{|c|c|}
\hline $\begin{array}{l}\text { XII } 27 \text { De nicticorace. Noctem amat, quia de } \\
\text { nocte volans cibum querit. } \\
\text { Nota qui male agit odit lucem }\end{array}$ & $\begin{array}{l}\text { B VII (= Y VII) Et tenebras amat magis quam lucem. Hic } \\
\text { figuram gerit populi Iudeorum. }\end{array}$ \\
\hline $\begin{array}{l}\text { XII } 34 \text { De turture. Loca solitaria diligit et eligit } \\
\text { consortiaque hominum valde fugit. } \\
\text { Nota de contemplativis }\end{array}$ & $\begin{array}{l}\text { B XXVIII Talis est enim sancta ecclesia, que postquam vidit } \\
\text { virum suum crucifixum [...] alio viro non coniungitur. } \\
\text { Y XLI Christofori eligunt in secreto habitare [...] qui } \\
\text { imitantur turturem Dominum nostrum Christum. }\end{array}$ \\
\hline $\begin{array}{l}\text { XVIII } 90 \text { De salamandra. Ab illa pelle fiunt } \\
\text { ligamina in lampadibus et lucernis, que nullo } \\
\text { incendio corrumpuntur. } \\
\text { Nota de sanctis qui in tribulationibus non } \\
\text { deficiunt }\end{array}$ & $\begin{array}{l}\text { B XxX Isti sunt iusti et mirabiles omnibus hominibus Dei, } \\
\text { sicut fuerunt in camino ignis ardentis. } \\
\text { Y XLV Quanto melius, qui secundum iustitiam extinguerunt } \\
\text { virtutem ignis. }\end{array}$ \\
\hline $\begin{array}{l}\text { XVIII } 115 \text { De vipera. Catuli, non expectantes } \\
\text { maturam nature solutionem, corrosis eius } \\
\text { lateribus cum matris interitu vi erumpunt. } \\
\text { Nota de illis qui destruunt ecclesiam vel } \\
\text { religionem }\end{array}$ & $\begin{array}{l}\text { Y XII Assimilavit ergo Salvator noster Phariseos vipere: sicut } \\
\text { hec generatio occidit patrem et matrem, sic et hic populus, } \\
\text { qui sine Deo est, patrem suum Ihesum Christum et matrem } \\
\text { terrestrem Hierusalem. }\end{array}$ \\
\hline
\end{tabular}

Comme on le voit aisément, l'objectif principal du DPR est de condamner les vices et de promouvoir les vertus, alors que le Physiologus était plutôt intéressé par des interprétations spirituelles et symboliques, portant sur des questions fondamentales de la religion chrétienne (la figure du Christ et du diable, la polémique avec les Juifs, le salut). Des consonances entre les deux textes émergent surtout à propos de la représentation des péchés (voir les chapitres De vipera, De nicticorace, De bubalo, De onocentauro) et de l'exigence d'une purification (voir De aquila, De kaladrio, De angue, De castore) ; on trouve aussi un cas de figuration christologique attachée à l'attitude d'un animal (De pellicano) et des allusions aux qualités propres des bons chrétiens (De turture, De salamandra), alors que la contradiction majeure se manifeste dans l'interprétation de la panthère et de la licorne, qui représentent le Christ dans le Physiologus et le diable dans le DPR. Bien que l'œuvre de Barthélemy ne constitue qu'un exemple de ce que le processus millénaire d'interprétation allégorique de l'animal pouvait donner à l'époque et dans un certain milieu, notre bref inventaire donne ainsi l'occasion de mesurer quelle persistance (et quelles évolutions) présentent au fil des siècles certaines inspirations symboliques qui ont trouvé dans la littérature liée au Physiologus une forme d'expression parmi les plus caractéristiques ${ }^{45}$. 


\section{Conclusion}

Le Physiologus est une source secondaire du DPR, en raison du nombre limité d'informations qu'il pouvait offrir, mais surtout du caractère "moderne" de ce dernier, qui se nourrit plus volontiers de sources nouvelles émanant d'Aristote et de la science arabe, récemment traduits en latin. Bien que peu nombreuses, les citations du DPR qui sont attribuées explicitement ou peuvent être rapportées à cet ouvrage ne sont cependant pas dépourvues d'intérêt; elles encouragent des enquêtes croisées contribuant tantôt à un premier retour sur la transmission du DPR, tantôt à la mise en lumière d'un épisode inédit de la postérité de cette "source complexe ». Un réexamen d'une partie de la tradition directe du DPR, accompagné d'une édition critique des reprises en question, constitue la prémisse pour aborder un ensemble de textes dont les relations ne sont pas toujours évidentes et ne rendent compte des notions que Barthélemy met sous l'autorité du Physiologus que s'ils sont combinés. L'héritage du Physiologus passe en réalité à travers des versions récentes «mises à jour " grâce à l'apport isidorien, qui connaissent un travail de réécriture et d'enrichissement ultérieurs et auxquelles s'ajoutent des notions déjà filtrées par une compilation sur la nature «concurrente» comme celle de l'Experimentator. Le nom du Physiologus joue ainsi le rôle de "collecteur » d'une pluralité de textes et d'approches de la nature, comme rémanence d'un temps plus ancien qu'il nous importe de réinventer.

\section{BIBLIOGRAPHIE}

\section{Éditions}

BARTHOLOMAEI ANGLICI De genuinis rerum coelestium, terrestrium et inferarum proprietatibus, Francofurti, anno MDCI (réimpr. 1609, 1964).

BARTHOLOMAEUS ANGLICUS, De proprietatibus rerum. Vol. 1. Introduction générale (VAN DEN ABEELE Baudouin), Prohemium (MEYER Heinz), Libri I-IV (TWOMEY Michael W., ROLING Bernd, LONG R. James) ; Vol. 6. Liber XVII (VENTURA Iolanda), Turnhout, Brepols, 2007.

CAHIER Charles, MARTIN Arthur, Mélanges d'archéologie, d'histoire et de littérature, Paris, PoussielgueRusand, vol. 2, 1851, p. 107-232 ; vol. 3, 1853, p. 204-285 ; vol. 4, 1856, p. 57-70.

CARMODY Francis J., Physiologus Latinus : éditions préliminaires, Versio B, Paris, Droz, 1939.

CARMODY Francis J., Physiologus Latinus Versio Y, Berkeley, University of California Press, 1941.

CLARK Willene B., A Medieval Book of Beasts. The Second-family Bestiary: Commentary, Art, Text and Translation, Woodbridge, The Boydell Press, 2006.

DEUS Janine, Der 'Experimentator'. Eine anonyme lateinische Naturenzyklopädie des frühen 13.

Jahrhunderts, Dissertation zur Erlangung der Würde des Doktors der Philosophie des Fachbereichs Geschichtswissenschaft, Hamburg, 1998. 
MANN Max F., Der Bestiaire divin des Guillaume le Clerc, Heilbronn, Henninger, 1888.

THOMAS CANTIMPRATENSIS, Liber de natura rerum, BOESE Helmut (éd.), Berlin - New York, De Gruyter, 1973.

VINCENTII BELLOVACENSIS Speculum quadruplex sive Speculum maius, Douai, 1624, 4 t. (réimpr. Graz 1964).

WHITE Cynthia, From the Ark to the Pulpit: An Edition and Translation of the "Transitional" Northumberland Bestiary (13th Century), Louvain-la-Neuve, Publications de l'Institut d'études médiévales, 2009.

\section{Travaux}

Bartholomaeus Anglicus, De proprietatibus rerum, Texte latin et réception vernaculaire - Lateinischer Text und volkssprachige Rezeption, VAN DEN ABEELE Baudouin, MEYER Heinz (éd.), Turnhout, Brepols, 2005 (notamment VAN DEN ABEELE Baudouin, MEYER Heinz, «État de l'édition du De proprietatibus rerum », p. 1-12 ; DRAELANTS Isabelle, «La science naturelle et ses sources chez Barthélemy l'Anglais et les encyclopédistes contemporains », p. 43-99 ; VAN DEN ABEELE Baudouin, « Barthélemy l'Anglais et Jean Corbechon : enquêtes sur le livre XII, De avibus », p. 245-266).

BAXTER Ron, Bestiaries and Their Users in the Middle Ages, Stroud, Sutton, 1998.

CARMODY Francis J., « De bestiis et aliis rebus and the Latin Physiologus », Speculum, 13, 1938, p. 153-159.

CASAPUlLo Rosa, « Sull'edizione di un testo mediolatino a tradizione sovrabbondante: il De proprietatibus rerum di Bartolomeo Anglico », Filologia Italiana, 9, 2012, p. 9-25.

CIPRIANI Mattia, «In dorso colorem habet inter viridem et ceruleum... Liber rerum e osservazione zoologica diretta nell'enciclopedia di Tommaso di Cantimpré », Reinardus, 29, 2017, p. 16-98.

CLARK Willene B., " Four Latin Bestiaries and De bestiis et aliis rebus », in Bestiaires médiévaux. Nouvelles perspectives sur les manuscrits et les traditions textuelles, VAN DEN ABEELE Baudouin (éd.), Louvain-la-Neuve, Publications de l'Institut d'études médiévales, 2005, p. 49-69.

DINES Ilya, « The Problem of the Transitional Family of Bestiaries », Reinardus, 24, 2012, p. 29-52. DRAELANTS Isabelle, FRUNZEANU Eduard, « Le savoir astronomique et ses sources dans le De mundo et corporibus celestibus de Barthélemy l'Anglais ", Rursus [En ligne], 11, 2017, mis en ligne le 22 décembre 2017, consulté le 13 mai 2019. URL : http://journals.openedition.org/rursus/1352. DOI : $10.4000 /$ rursus.1352.

Encyclopédie médiévale et langues européennes. Réception et diffusion du De proprietatibus rerum de Barthélemy l'Anglais dans les langues vernaculaires, Ducos Joëlle (éd.), Paris, Honoré Champion, 2014.

KAY Sarah, «The English Bestiary, the Continental Physiologus, and the Intersections between them », Medium Aevum, 85, 2016, p. 118-142.

KUHRY Emmanuelle, « Dictionnaires, distinctions, recueils de propriétés en milieu cistercien. Outils pour la prédication, sources pour l'étude de la nature », in Les cisterciens et la transmission des textes (XII ${ }^{e}$-XVIII ${ }^{e}$ siècles), FALMAGNE Thomas, STUTZMANN Dominique, TURCAN-VERKERK AnneMarie, avec la collaboration de GANDIL Pierre (éd.), Turnhout, Brepols, 2018, p. 285-337. KUHRY Emmanuelle, «Les chapitres sur les poissons et créatures aquatiques dans la tradition manuscrite du Physiologus latinus et des bestiaires latins, et leur réception dans quelques 
encyclopédies médiévales ", in Inter litteras et scientias. Recueil d'études en hommage à Catherine Jacquemard, GAUVIN Brigitte, LUCAS-AVENEL Marie-Agnès (éd.), Caen, Presses universitaires, 2019, p. 117-150.

LIBROVÁ Bohdana, «Le renard dans le cubiculum taxi : les avatars d'un exemplum et le symbolisme du blaireau », Le Moyen Âge, 109, 2003, p. 79-111.

LONCKE Jérémy, « Approche comparative de la diffusion et de la transmission des différents états manuscrits du De Proprietatibus Rerum de Barthélemy l'Anglais ", in Une lumière venue d'ailleurs : héritages et ouvertures dans les encyclopédies d'Orient et d'Occident au Moyen Âge. Actes du colloque de Louvain-la-Neuve (19-21 mai 2005), DE CALLATAŸ Godefroid, VAN DEN ABEELE Baudouin (éd.), Louvain-laNeuve, Brepols, 2008, p. 177-198.

MCCULLOCH Florence, Mediaeval Latin and French Bestiaries, Chapel Hill (NC), University of North Carolina Press, 1962.

MEYER Heinz, Die Enzyklopädie des Bartholomäus Anglicus. Untersuchungen zur Überlieferungs- und Rezeptionsgeschichte von 'De proprietatibus rerum', München, Fink, 2000.

STURLESE Loris, « Florilegi filosofici ed enciclopedie in Germania nella prima metà del Duecento. Gli scritti di Arnoldo di Sassonia e di Bartolomeo l'Inglese e la diffusione della scienza araba e aristotelica nella cultura tedesca », Giornale critico della filosofia italiana, 69, 1990, p. 293-319. VAN DEN ABEELE Baudouin, MEYER Heinz, RIBÉMONT Bernard, « Éditer l'encyclopédie de Barthélemy l'Anglais. Vers une édition bilingue du De proprietatibus rerum ", Cahiers de recherches médiévales et humanistes, 6, 1999, p. 7-18.

VAN DEN ABEELE Baudouin, «À la recherche de l'Experimentator de Thomas de Cantimpré », in Expertus sum. L'expérience par les sens dans la philosophie naturelle médiévale. Actes du colloque international de Pont-à-Mousson (5-7 février 2009), BÉNATOUÏL Thomas, DRAELANTS Isabelle (éd.), Firenze, SISMEL-Edizioni del Galluzzo, 2011, p. 41-65.

VAN DEN ABEELE Baudouin, « Simbolismo sui margini. Le moralizzazioni del De proprietatibus rerum di Bartolomeo Anglico », in Simbolismo animale e letteratura, FARACI Dora (éd.), Manziana, Vecchiarelli, 2003, p. 159-183.

VENTURA Iolanda, « Towards a Critical Edition of Bartholomew the Englishman's De proprietatibus rerum: Editorial Principles, Goals, and Open Paths », W-mail : Werkgroep Middelnederlandse Artesliteratuur, 11, 2010, p. 18-32.

\section{Ressources en ligne}

SOURCENCYME - Sources des Encyclopédies Médiévales, corpus annoté (http://sourcencyme.irht.cnrs.fr). LONCKE Jérémy, Bartholomaeus Anglicus - De proprietatibus rerum. Exploitation des notes marginales (http://www.lejer.be/marginalia/intro.htm).

\section{NOTES}

1. Je remercie vivement Isabelle Draelants de m'avoir invitée à participer à la journée d'étude $L a$ réception du Physiologus dans les encyclopédies médiévales, qui s'est tenue à l'IRHT (CNRS - Paris) le 14 juin 2017, ainsi que d'avoir lu et corrigé attentivement la présente version écrite de ma communication. Je suis également reconnaissante à Emmanuelle Kuhry, qui m’a fourni plusieurs 
matériaux et indications sans lesquels cet article, qu'elle a relu avec patience, n'aurait jamais pu atteindre sa forme définitive.

2. Pour une présentation synthétique du personnage et de son ouvrage, voir l'introduction au volume I de l'édition critique du DPR en cours de publication chez Brepols (BARTHOLOMAEus ANGLICUS, De proprietatibus rerum. Vol. 1. Introduction générale (VAN DEN ABEELE B.), Prohemium (MEYER H.), Libri I-IV (TWOMEY M. W., ROLING B., LONG R. J.), Turnhout, 2007, notamment p. 3-16 et l'ample bibliographie dressée en p. 35-43). Quant à la chronologie de composition du DPR, les chercheurs qui préparent actuellement cette édition confirment les dates acceptées par l'historiographie (voir notamment STURLESE L., «Florilegi filosofici ed enciclopedie in Germania nella prima metà del Duecento. Gli scritti di Arnoldo di Sassonia e di Bartolomeo l'Inglese e la diffusione della scienza araba e aristotelica nella cultura tedesca ", Giornale critico della filosofia italiana, 69, 1990, p. 293-319). En même temps, les études parues sur les sources utilisées par Barthélemy permettent de dire que les divers livres du DPR ont été composés ou enrichis à différents moments, car une partie de leurs sources n'était disponible qu'à Paris, alors que d'autres ont commencé à circuler seulement après $c .1230$ (pour ne citer qu'un exemple très récent, voir l'enquête sur les sources astronomiques du livre VIII de DRAELANTS I., FRUNZEANU E., « Le savoir astronomique et ses sources dans le De mundo et corporibus celestibus de Barthélemy l'Anglais ", Rursus [En ligne], 11, 2017, mis en ligne le 22 décembre 2017, consulté le 13 mai 2019. URL: http://journals.openedition.org/rursus/1352. DOI : 10.4000/rursus.1352).

3. MEYER H., Die Enzyklopädie des Bartholomäus Anglicus. Untersuchungen zur Überlieferungs- und Rezeptionsgeschichte von 'De proprietatibus rerum', München, Fink, 2000 ; voir aussi LONCKE J., «Approche comparative de la diffusion et de la transmission des différents états manuscrits du De Proprietatibus Rerum de Barthélemy l'Anglais", in Une lumière venue d'ailleurs: héritages et ouvertures dans les encyclopédies d'Orient et d'Occident au Moyen Âge. Actes du colloque de Louvain-laNeuve (19-21 mai 2005), DE CALLATAŸ G., VAN DEN ABEELE B. (éd.), Louvain-la-Neuve, Brepols, 2008, p. 177-198. B. Van den Abeele a refait le compte des témoins de la forme originaire du DPR dans l'introduction générale de BARTHOLOMAEUS ANGLICUS, De proprietatibus rerum. Vol. 1, 2007 : 18-21.

4. Outre le volume I, déjà cité à la note 1 , voir BARTHOLOMAEUS ANGLICUS, De proprietatibus rerum. Vol. 6. Liber XVII (VENTURA I.), Turnhout, Brepols, 2007 et BARTHOLOMAEUS ANGLICUS, De proprietatibus rerum. Vol. 3. Liber VI (LIDAKA J., VENTURA I., ZARRA G.), Liber VIII (DRAELANTS I., FRUNZEANU E., VENTURA I.), Liber IX (SUNTRUP R.), Turnhout, Brepols, sous presse.

5. Voir surtout les contributions recueillies dans Bartholomaeus Anglicus, De proprietatibus rerum, Texte latin et réception vernaculaire - Lateinischer Text und volkssprachige Rezeption, VAN DEN ABEELE B., MEYER H. (éd.), Turnhout, Brepols, 2005 et Encyclopédie médiévale et langues européennes. Réception et diffusion du De proprietatibus rerum de Barthélemy l'Anglais dans les langues vernaculaires, Ducos J. (éd.), Paris, Honoré Champion, 2014.

6. BARTHOLOMAEI ANGLICI De genuinis rerum coelestium, terrestrium et inferarum proprietatibus, Francofurti, anno MDCI (réimpr. 1609, 1964).

7. Voir BaRtholomaeus ANGlicus, De proprietatibus rerum. Vol. 1, 2007 : 22-33; VAN DEN ABEele B., MEYER H., RIBÉMONT B., «Éditer l'encyclopédie de Barthélemy l'Anglais. Vers une édition bilingue du De proprietatibus rerum », Cahiers de recherches médiévales et humanistes, 6, 1999, p. 7-18 ; VAN DEN ABEELE B., MEYER H., «État de l'édition du De proprietatibus rerum ", in Bartholomaeus Anglicus, De proprietatibus rerum, 2005 : 1-12; VENTURA I., «Towards a Critical Edition of Bartholomew the Englishman's De proprietatibus rerum: Editorial Principles, Goals, and Open Paths ", W-mail: Werkgroep Middelnederlandse Artesliteratuur, 11, 2010, p. 18-32.

8. CASAPULLO R., «Sull'edizione di un testo mediolatino a tradizione sovrabbondante: il De proprietatibus rerum di Bartolomeo Anglico », Filologia Italiana, 9, 2012, p. 9-25.

9. Plinius Anglicus, Plinius Gallicus et alii. Inchieste filologiche (e non solo) tra la Naturalis Historia di Plinio il Vecchio e suoi epigoni tardomedievali (Plinius Anglicus, Plinius Gallicus et alii. Enquêtes 
philologiques (et pas uniquement) entre la Naturalis Historia de Pline l'Ancien et ses épigones du Moyen Âge tardif), mémoire inédit soutenu à l'Université de Pise le 12 octobre 2016 sous la direction de E. Stagni et A. Zago.

10. Les apparats que je constitue enregistrent toutes les leçons significatives des vingt et un manuscrits collationnés, en omettant celles propres à un seul témoin et/ou insignifiants. Je souligne que je ne dispose pas toujours de tous les témoins, parce que $B$ a perdu quasiment tout le livre XII et d'autres codices résument parfois le texte du DPR à travers des omissions et des réécritures. La tendance est particulièrement évidente dans $F$ et $J$ et attestée dans $H$ et $L$, mais comme elle n'a jamais été signalée, ces manuscrits sont encore considérés comme des témoins complets du texte.

11. Un examen plus approfondi de la tradition manuscrite du DPR à la lumière de l'une de ses sources majeures, à savoir la Naturalis historia de Pline l'Ancien, se trouve dans LONATI E., «Bartolomeo Anglico, the English Pliny? », Archivum Latinitatis Medii Aevi, 76, 2018, p. 223-278.

12. Il est impossible d'entrer dans le détail des sources dont Barthélemy se sert pour rédiger chaque section de son texte ; un examen extrêmement utile de cet éventail de sources, conduit en comparaison avec les plus importants ouvrages encyclopédiques de l'époque, se trouve dans DRAELANTS I., « La science naturelle et ses sources chez Barthélemy l'Anglais et les encyclopédistes contemporains ", in Bartholomaeus Anglicus, De proprietatibus rerum, 2005 : 43-99.

13. Cette perspective se révèle clairement dans certains livres du $D P R$, alors qu'elle est moins évidente dans d'autres. Une analyse de la double attitude, qui porte Barthélemy à privilégier presqu'exclusivement les oiseaux bibliques dans le livre XII, mais à élargir l'éventail des créatures décrites dans le livre XVIII bien au-delà de celles mentionnées par la Bible, se trouve dans VAN DEN ABEELE B., "Barthélemy l'Anglais et Jean Corbechon : enquêtes sur le livre XII, De avibus ", in Bartholomaeus Anglicus, De proprietatibus rerum, 2005 : 245-266.

14. Pour un traitement détaillé de la question voir KAY S., "The English Bestiary, the Continental Physiologus, and the Intersections between them ", Medium Aevum, 85, 2016, p. 118-142 et KUHRY E., "Les chapitres sur les poissons et créatures aquatiques dans la tradition manuscrite du Physiologus latinus et des bestiaires latins, et leur réception dans quelques encyclopédies médiévales ", in Inter litteras et scientias. Recueil d'études en hommage à Catherine Jacquemard, GAUVIN B., LUCAS-AVENEL M.-A. (éd.), Caen, Presses universitaires, 2019, p. 117-150 (je tiens à remercier l'auteure, qui m'a permis d'en lire la version achevée avant publication). Au tout début de la seconde contribution se trouve une petite récapitulation des versions du Physiologus, apportant quelques corrections par rapport aux filiations avancées auparavant ; je me suis d'ailleurs fondée sur la liste des manuscrits qu'elle propose pour choisir les témoins à consulter. Si une première mise au point des différentes étapes de diffusion du Physiologus était déjà dans MCCULLOCH F., Mediaeval Latin and French Bestiaries, Chapel Hill (NC), University of North Carolina Press, 1962, un bilan plus approfondi et novateur est en cours de préparation par A. Zucker et E. Kuhry ; l'article d'E. Kuhry dans ce même numéro de RursuSpicae consacré au Physiologus en livre les principaux acquis.

15. Pour l'édition de $A, B$ et $C$, voir respectivement CAHIER C., MARTIN A., Mélanges d'archéologie, d'histoire et de littérature, Paris, Poussielgue-Rusand, vol. 3, 1853, p. 204-285 ; vol. 4, 1856, p. 57-70 ; vol. 2, 1851, p. 107-232. Pour la version B, il faut cependant préférer l'édition publiée par CARMODY F. J., Physiologus Latinus : éditions préliminaires, Versio B, Paris, Droz, 1939.

16. CARMODY F. J., Physiologus Latinus Versio Y, Berkeley, University of California Press, 1941, p. 95-134.

17. MANN M. F., Der Bestiaire divin des Guillaume le Clerc, Heilbronn, Henninger, 1888, p. 37-73 (édition du ms. London, British Library, Royal 2.C.XII) ; j'ai consulté exempli gratia le témoin Città del Vaticano, Biblioteca Apostolica Vaticana, Pal. lat. 1074 ( $\mathrm{X}^{\mathrm{e}} / \mathrm{XI}^{\mathrm{e}}$ siècle ; https://digi.vatlib.it/ view/MSS_Pal.lat.1074). 
18. Transmis (au moins partiallement) par quatre témoins, parmi lesquels j'utilise Cambridge, Corpus Christi College, 22 (autour de 1150-1170; https://parker.stanford.edu/parker/catalog/ xd956wx5361) ; les autres sont : Cambridge, University Library, Mm.6.15 ; London, British Library, Stowe 1067 ; New Haven, Yale, Beinecke 851.

19. CLARK W. B., A Medieval Book of Beasts. The Second-family Bestiary: Commentary, Art, Text and Translation, Woodbridge, The Boydell Press, 2006. J'ai vérifié exempli gratia le ms. London, British Library, Harl. 3244 (autour de 1230-1250; http://www.bl.uk/manuscripts/Viewer.aspx? ref=harley_ms_3244_f036r).

20. WHITE C., From the Ark to the Pulpit: An Edition and Translation of the "Transitional" Northumberland Bestiary (13th Century), Louvain-la-Neuve, Publications de l'Institut d'études médiévales, 2009. J'ai consulté exempli gratia le ms. London, British Library, Royal 12.C.XIX (autour de 1200 ; http:// www.bl.uk/manuscripts/Viewer.aspx?ref=royal_ms_12_c_xix_f006r).

21. Voir l'étude du chapitre sur la sirène dans KUHRY, «Les chapitres sur les poissons », 2019 ; le texte est vérifié sur Chalon-sur-Saône, Bibliothèque municipale, 14 (autour de 1280 ; http:// bvmm.irht.cnrs.fr/consult/consult.php?reproductionId=12742) et Paris, Bibliothèque nationale de France, lat. 14429 (XIII ${ }^{\mathrm{e}}$ siècle ; https://gallica.bnf.fr/ark:/12148/btv1b10033822c/f113.image).

22. Le buffle n'apparaît que dans des versions tardives du Physiologus, d'après une notice isidorienne ; le texte en question concerne en fait l'antilope (autolops).

23. Ces informations sont à détecter dans deux chapitres différents des bestiaires liés à B-Is. Comme KUHRY, «Les chapitres sur les poissons», 2019 le montre, le crocodile ne fait l'objet d'aucun chapitre dans $Y, B, C$ et $B$-Is, qui ajoute les informations à ce sujet à la fin du passage sur l'hydrus. Ce chapitre représente donc un ajout propre à la version H-B-Is, où il suit immédiatement le chapitre sur l'hydrus, du même que dans $H$. Dans $S$ le chapitre sur le crocodile fait son apparition parmi les quadrupèdes et, sous forme partielle, aussi dans le catalogue de poissons isidorien ajouté au noyau ancien; le chapitre sur l'hydrus, suivi par un chapitre sur l'hydra, où se trouve la notice de la lutte avec le crocodile, fait partie du catalogue des serpents. $T$, enfin, distribue les informations entre deux chapitres consécutifs, sur l'hydrus et sur l'hydra, placés parmi les quadrupèdes, mais il répète la narration de la lutte, avec des variations, dans le catalogue des serpents.

24. Proche de B-Is est $H$ : Cum comederit et satiaverit se de universis venationibus, recipit se in speluncam suam, ponensque se dormit [...] Solus autem draco, cum audierit vocem eius, abscondit se sub terrenis speluncis; ibi autem non ferens vim odoris eius in semetipso contractus obstupescit.

25. Comme E. Kuhry le montre, selon le témoin, $T$ peut s'aligner sur l'une ou sur l'autre version pour les deux éléments.

26. DEUS J., Der 'Experimentator'. Eine anonyme lateinische Naturenzyklopädie des frühen 13. Jahrhunderts, Dissertation zur Erlangung der Würde des Doktors der Philosophie des Fachbereichs Geschichtswissenschaft, Hamburg, 1998. La première version (Fassung I) est présente dans les codices Berlin, Staatsbibliothek Preussischer Kulturbesitz, Hamilton 277; Pisa, Biblioteca Cateriniana, 30; Roma, Biblioteca Angelica, 1038 (R.7.1); Stuttgart, Württembergische Landesbibliothek, med. et phys. $2^{\circ} 24$; la Fassung II par les manuscrits Chambéry, Bibliothèque municipale, 30 ; London, British Library, Sloane 3167; Madrid, Biblioteca Nacional, 12803; Milano, Biblioteca Ambrosiana, A 147 sup. ; Soissons, Bibliothèque municipale, 26 ; la version abrégée ne se trouve que dans le manuscrit Wolfenbüttel, Herzog-August Bibliothek, 8.8 Aug. $4^{\circ}$. J. Deus a édité intégralement cette dernière et a fourni une édition partielle de la Fassung $I$.

27. MEYER, Die Enzyklopädie des Bartholomäus Anglicus, 2000 : 149-163 ; VAN DEN ABEELE B., «À la recherche de l'Experimentator de Thomas de Cantimpré ", in Expertus sum. L'expérience par les sens dans la philosophie naturelle médiévale. Actes du colloque international de Pont-à-Mousson (5-7 février 2009), BÉNATOUïL Th., DRAELANTS I. (éd.), Firenze, SISMEL-Edizioni del Galluzzo, 2011, p. 41-65. Voir DRAELANTS, FRUNZEANU, «Le savoir astronomique et ses sources dans le De mundo », 2017 pour un 
bilan sur la question, une liste mise à jour des témoins de cet ouvrage et une série d'exemples où Barthélemy utilise l'Experimentator édité par J. Deus.

28. Cette information pourrait rappeler le contenu de la seconde partie du De onagro (à partir de Sagax), qui n'est pas dans le Physiologus; elle est cependant trop rapide et générique pour que Barthélemy puisse l'avoir reprise de l'Experimentator.

29. La Fassung II de l'Experimentator, d'après le ms. Chambéry, B.M., 30, ajoute cette information, qui est déjà dans les bestiaires : Lupi habentes fetus non agunt predas de prope, ne incitati pastores fetus inveniant.

30. Le texte de $H$ est identique au niveau du contenu, mais légèrement différent en ce qui concerne la formulation.

31. Plus loin dans le texte, le témoin Paris, B.n.F., lat. 14429 répète la description de cet oiseau, en changeant la formulation du passage en question (Totus albus est, nullam partem nigri in se habens, cuius interior fimus...).

32. La version $H$ ne semble pas transmettre cette description, alors que la formulation de H-B-Is est assez éloignée.

33. Voir aussi, pour ce seul cas, le bestiaire H-B-Is (cum senuerit ita ut videre vel vivere nequeat, pulli eius evellunt invalidas eius pennas [...] donec recrescant penne eius et renovata sit et videat sicut et ipsi clare).

34. Le texte de $H$ est plus éloigné (cum senuerit et volare non possit, filii eius ad eam veniunt et pennas vetustissimas a corpore ipsius evellunt [...] donec iterum penne nove recrescant. Cibis sustentant [...] donec sicut antea sumptis viribus volare possit) et $S$ (quando viderit parentes suos senuisse et caligasse oculos eorum, evellit plumas eorum [...] et renovantur parentes ipsius).

35. Il faut quand même remarquer que déjà dans la tradition de la Fassung I éditée par J. Deus, certains témoins portent l'attribution trompeuse au Philosophus pour les extraits sur le phénix et le kaladrius.

36. KUHRY E., « Dictionnaires, distinctions, recueils de propriétés en milieu cistercien. Outils pour la prédication, sources pour l'étude de la nature ", in Les cisterciens et la transmission des textes (XII ${ }^{e}$-XVIII ${ }^{e}$ siècles), FALMAGNE Th., STUTZMANN D., TURCAN-VERKERK A.-M. avec la collaboration de GANDIL P. (éd.), Turnhout, Brepols, 2018, p. 285-337.

37. Il a été considéré à tort comme une forme abrégée du DPR, mais, selon E. Kuhry, les deux textes remonteraient plutôt à un amas d'informations sur les caractéristiques physiques des objets naturels circulant ensemble, sous diverses formes, dès le XII ${ }^{\mathrm{e}}$ siècle.

38. Terminée après 1240 et remaniée à plusieurs reprises, cette encyclopédie a été éditée pour la première fois par Helmut Boese (THOMAS CANTIMPRATENSIS, Liber de natura rerum, BOESE H. (éd.), Berlin - New York, De Gruyter, 1973) ; une nouvelle édition et une étude globale de ses sources sont en cours d'achèvement par Mattia Cipriani, qui a consacré à ce sujet sa thèse de doctorat, soutenue en 2014 (La place de Thomas de Cantimpré dans l'encyclopédisme médiéval: les sources du Liber de natura rerum). Son édition critique provisoire du texte du Liber, avec l'identification de la plupart des sources utilisées par Thomas, a paru en ligne au cours de 2017 dans le corpus SOURCENCYME (http://sourcencyme.irht.cnrs.fr) de l'Atelier Vincent de Beauvais.

39. Composée entre 1244 et 1260, cette énorme encyclopédie en trois volumes (Speculum historiale, naturale, doctrinale) a été consultée dans la dernière des éditions anciennes, encore de référence (VincentiI Bellovacensis Speculum quadruplex sive Speculum maius, Douai, 1624, 4 t., réimpr. Graz 1964); son texte est intégralement mis à disposition en ligne dans le corpus SOURCENCYME.

40. À propos de cette source, cf. CIPRIANI M., «In dorso colorem habet inter viridem et ceruleum... Liber rerum e osservazione zoologica diretta nell'enciclopedia di Tommaso di Cantimpré », Reinardus, 29, 2017, p. 16-98 ; p. 45, n. 92 l'auteur signale déjà le rapprochement entre les textes de Thomas et Barthélemy. 
41. SN XIX 110 Plinius ubi supra. [...] Fertur autem huic vulpes quodammodo esse contraria. Vulpes enim nunquam sibi foveam parat, sed foveam taxi per dolum occupat. Cum enim eum exisse inde scit, ad introitum vadit et ibi stercorat, sicque taxo propter immunditiam locum deserente vulpes eum occupat. [...] Physiologus. Taxi mansiones sibi subterraneas labore parant et unum taxum eligunt, terre pedibus suis effosse vectorem; itaque supinatus quidem extensisque cruribus et erectis supra ventrem suum terra effossa accumulatur oneraturque, per pedes ab aliis exportatur. Totiensque hoc faciunt quousque domus latitudo habitatoribus sufficere possit.

42. Pour une analyse de la figure du blaireau et de ses évolutions, voir LIBRová B., «Le renard dans le cubiculum taxi: les avatars d'un exemplum et le symbolisme du blaireau ", Le Moyen Âge, 109, 2003, p. 79-111 (https://www.cairn.info/revue-le-moyen-age-2003-1-page-79.htm), qui m'a été gentiment signalé par E. Kuhry. Quant à l'ouvrage d'Alexandre Neckam, voir l'édition de Th. Wright, London, $1863: 207$.

43. Pour une présentation synthétique de la question voir BARTHOLOMAEUS ANGLicus, De proprietatibus rerum. Vol. 1, 2007 : 10-11, 32-33. J. Loncke, qui s'est penché longuement sur l'étude de cette caractéristique de la tradition du DPR, a de son côté mis en ligne un corpus de notes marginales interrogeable et visant à une expansion progressive, fondé sur les livres XI à XVII pour les témoins A B (Bartholomaeus Anglicus - De proprietatibus rerum. Exploitation des notes marginales). Voir aussi VAN DEN ABEELE B., «Simbolismo sui margini. Le moralizzazioni del De proprietatibus rerum di Bartolomeo Anglico ", in Simbolismo animale e letteratura, FARACI D. (éd.), Manziana, Vecchiarelli, 2003, p. 159-183.

44. Une bonne partie des exemplaires tardifs que j'ai ajoutés aux cinq manuscrits de base présente des notes dans la forme dite "standard ", c'est-à-dire qu'ils offrent un corpus assez constant, bien que certains suppriment ou raccourcissent sporadiquement quelques notes. D'autres témoins comme $B, C, D$ ont des notes supplémentaires par rapport aux autres (par ex., ils en ont aussi au livre XVI) et modifient les contenus de certaines moralisations ainsi que leur articulation tout au long du texte. Plus précisement, dans notre corpus les témoins qui portent des notes sont $A, B, C, D, E, K, L, M, N, O, P, Q, S, T, U$, mais, comme ils ne sont pas tous disponibles pour chaque passage, ni toujours superposables, je présente dans les tableaux suivants seulement les notes marginales principales dans leur forme la plus typique et complète.

45. E. Kuhry me suggère qu'il serait peut-être intéressant de comparer les notes du DPR avec les interprétations contenues dans les recueils de distinctions bibliques, à savoir des répertoires exploités au XIII ${ }^{\mathrm{e}}$ siècle par les prédicateurs, fournissant pour une série de termes tirés des Écritures un (ou plusieurs) des quatre sens (littéral, allégorique etc.) et un (ou plusieurs) exemples bibliques associés (voir KUHRY, "Dictionnaires, distinctions, recueils de propriétés", 2018 : 289-290). Entre la fin du XII ${ }^{\mathrm{e}}$ siècle et le début du XIII ${ }^{\mathrm{e}}$ furent en effet produites des collections comme la Summa Abel de Pierre le Chantre, la Summa «Quot modis » d'Alain de Lille, la Summa super Psalterium de Prévostin de Crémone, les Distinctiones super Psalterium de Pierre de Poitiers, les Distinctiones d'Etienne Langton, l'Angelus parfois attribué à Garnier de Rochefort, l'Alphabetum in artem sermocinandi de Pierre de Capoue. Selon les informations qu'E. Kuhry m'a très gentiment fournies, aucun rapprochement avec le DPR ne semble cependant émerger des recueils de Pierre le Chantre, Alain de Lille et Garnier de Rochefort et aucun indice ne permet pour l'instant de mettre en relation le texte de Barthélemy avec d'autres collections, au moins à mes yeux de non-spécialiste. 


\section{RÉSUMÉS}

Cet article réfléchit sur la présence du Physiologus dans le De proprietatibus rerum (DPR), l'encyclopédie composée par le franciscain Barthélemy l'Anglais autour de 1230-1248 entre Paris et Magdebourg. Après une présentation du DPR et de quelques questions méthodologiques à prendre en compte pour aborder sa tradition manuscrite, il propose une édition critique provisoire des passages liés au Physiologus. Sur cette base sont développées des observations visant à identifier la source précise de Barthélemy, en analysant les formes principales du Physiologus diffusées à l'époque et quelques compilations qui auraient pu jouer le rôle de sources intermédiaires.

This article aims to outline the Physiologus's presence in the De proprietatibus rerum (DPR), the encyclopaedia composed by the Franciscan Bartholomew the Englishman around 1230-48 between Paris and Magdeburg. After a short presentation of the DPR and of some methodological problems concerning the study of its manuscript tradition, we offer a provisional critical edition of all the quotations related to the Physiologus. On this basis, we develop some reflections to identify their source, analysing the Physiologus's versions known at the time and some other works, which may have been used as an intermediary between the original text and the DPR.

\section{INDEX}

Mots-clés : Description zoologique, moralisation, tradition manuscrite, Barthélemy l'Anglais, Physiologus

Keywords : Zoological description, Moralization, Manuscript tradition, Bartholomew the Englishman, Physiologus

\section{AUTEUR}

\section{ELISA LONATI}

Ancienne élève de la Scuola Normale Superiore et de l'Université de Pise, depuis octobre 2017 Elisa Lonati est doctorante contractuelle de l'École Pratique des Hautes Études, en cotutelle avec la Scuola Normale Superiore de Pise ; sa thèse porte sur l'édition, l'étude des sources et de la réception du Chronicon du cistercien Hélinand de Froidmont. 